\title{
Estimates of the Laplacian Spectrum and Bounds of Topological Invariants for Riemannian Manifolds with Boundary
}

\author{
Luca Sabatini
}

\begin{abstract}
We set out to obtain estimates of the Laplacian Spectrum of Riemannian manifolds with non-empty boundary. This was achieved using standard doubled manifold techniques. In simple terms, we pasted two copies of the same manifold along their common boundary thereby obtaining a Riemannian manifold with empty boundary and with a $C^{0}$-metric. This made it possible to adapt some estimates of the spectrum dependent on the volume or genus of the manifold as calculated in recent years by several authors. In order to extend further estimates that depend on the curvature, it is necessary to regularize the metric of the doubled manifold so that the new metric is isometric to that of each copy and such that the curvature has a finite lower bound. Controlling the curvature in this way also makes estimates of topological invariants available.
\end{abstract}

\section{Introduction}

It is well-known that an explicit calculation of the Laplacian Spectrum of a Riemannian manifold is, in the broadest terms, impossible. Among the classes of compact Riemannian manifolds, few allow an explicit calculation of the spectrum, which usually occurs in the presence of high levels of symmetry (e.g.

Key Words: Double of a manifold; Laplacian; Topological invariants; Regularized metric with control of curvature.

2010 Mathematics Subject Classification: Primary 35P15 53C20; Secondary 53C21 $58 \mathrm{C} 40$.

Received: 22.08.2018

Accepted: 12.09 .2018 
ESTIMATES OF THE LAPLACIAN SPECTRUM AND BOUNDS OF TOPOLOGICAL INVARIANTS FOR RIEMANNIAN MANIFOLDS WITH BOUNDARY.

the sphere $\left(\mathbb{S}^{n} ;\right.$ can $\left.)\right)$ or in some flat manifolds such as the torus $\left(T^{n} ;\right.$ flat $)$. This is the reason why it is sometimes sufficient to obtain some information by calculating, when possible, a lower and/or upper bound of each eigenvalue with particular attention to the first non zero one. Latterly, several authors contributed a number of the estimates for compact connected Riemmanian manifolds with empty or non-empty boundaries. M. Berger, P. Gauduchon, and E. Mazet have proposed a broad view on this subject in their famous and pioneering book ([1] (1987)). All of these estimates depend on the geometry of the manifold via the diameter, the volume and the sectional curvature. Some of these estimates require no special assumptions regarding the lower bound of Ricci curvature, however in others, this lower bound is necessary. In this paper we extend these estimates to compact connected Riemannian manifolds with non-empty boundary. These were obtained using standard doubled manifold techniques. By pasting two isometric copies of the same manifold along their common boundary we get a compact connected manifold $(M \sharp M ; g \sharp g)$ with empty boundary. The metric of the doubled manifold is naturally of $C^{0}$-class and $C^{0}$-limit of $C^{\infty}$-metric defined on it (see Lemma 2.1) which makes it possible to directly apply all results that do not require assumptions about the curvature (see Section 3) to the doubled manifold. For other estimates, fixing a lower bound of Ricci curvature, the highest level of metric regularity in the doubled manifold is necessary. Although if it is always possible to get a $C^{\infty}$-metric on $M \sharp M$ via a regularization, the necessary and inalienable lower bound of Ricci curvature might be lost in the doubled manifold in the neighbourhod of the common boundary. If the boundary $\partial M$ is convex (see Definition 4.1) no particular problems arise in the doubled manifold because:

Main Theorem A: Let $(M, \partial M)$ be a Riemannian manifold with convex boundary, then there exists a sequence of $C^{\infty}$-metrics $\left\{g_{k}\right\}_{k \in \mathbb{N}}$ on $M \sharp M$, converging in the $C^{0}$ topology to $g \sharp g$ and preserving the lower bound of the sectional curvature $\sigma_{g}$, i.e.

$$
\min \left(\sigma_{g_{k}}\right) \geq\left(\sigma_{g}\right)
$$

where the minimum is achieved on all the 2-planes tangent to $M$.

If the boundary is not convex, regularization is still possible, however the new metric of $M \sharp M$ depends on the geometry and the curvature bounds of the boundary $\partial M$. This is proved in the second main result regarding the modified metric of the doubled manifold:

Main Theorem B: Let $(M, \partial M, g)$ be a compact Riemannian manifold with sectional curvature bounded from below $\left(\sigma_{g} \geq-k^{2}\right)$ for some real $k$, with the 
ESTIMATES OF THE LAPLACIAN SPECTRUM AND BOUNDS OF

TOPOLOGICAL INVARIANTS FOR RIEMANNIAN MANIFOLDS WITH

BOUNDARY.

non-empty boundary whose injectivity radius and main curvature are such that

$$
\operatorname{inj}_{\partial M} \geq a, \quad h_{\partial M} \leq \eta
$$

for some real numbers $a>0$ and $\eta \geq 0$; then there exists a metric $\tilde{g}$ on $(M, \partial M)$ such that $\tilde{g} \sharp \tilde{g}$ is $C^{\infty}$ on $M \sharp M$ and such that

$$
\begin{gathered}
\left(\frac{\sinh \left(\frac{k a}{4}\right)}{\sinh \left(\frac{k a}{2}\right)}\right)^{2} \cdot g \leq \tilde{g} \leq \\
{\left[\cosh \left(\frac{k a}{4}\right)+\sup \left(1, \frac{\eta}{k}\right) \cdot \sinh \left(\frac{k a}{4}\right)\right]^{2 n-2} \cdot\left(\frac{\sinh \left(\frac{k a}{2}\right)}{\sinh \left(\frac{k a}{4}\right)}\right)^{2 n-4} \cdot g}
\end{gathered}
$$

and

$$
\sigma_{\tilde{g}} \geq-\frac{4 k^{2}}{\tanh ^{2}\left(\frac{k a}{4}\right)}
$$

These theorems make it possible to extend all results requiring a lower bound of Ricci curvature to compact connected Riemannian manifolds with non-empty boundary, separating those for which the boundary of $(M, \partial M, g)$ is convex (Section 4.1) and thereafter to those for which the boundary is not (Section 4.2). It is clear that much of the present article is to prove the two Main Theorems A and B that, despite their complexity, only play a functional role. The proof is shifted to the end of the text (Section 6) so as not to interrupt the presentation of the results concerning the estimates of the Laplacian spectrum and the bounds of topological invariants.

The knowledge of the spectrum of a Riemmanian manifold has not only theoretical importance but also has many potential uses: it is well known that the free oscillation frequencies of elastic bodies are strictly dependent on the shape and (obviously) on the material from which the body is made. The structural theories of elastic bodies enable the modelling of an elastic structural element such a Riemmanian manifold with suitable metric, depending on the material. See e.g. [13] on how to use the present results to get informations about vibration frequencies of linear elastic membranes. 
ESTIMATES OF THE LAPLACIAN SPECTRUM AND BOUNDS OF

TOPOLOGICAL INVARIANTS FOR RIEMANNIAN MANIFOLDS WITH

BOUNDARY.

\section{The double of the manifold}

\subsection{Definition of the double of a manifold $(M \sharp M, g \sharp g)$}

Let $(M, g)$ be a Riemannian manifold with compact and differentiable boundary $\partial M$; from the disjoint union $M_{1} \amalg M_{2}$ of two copies of the manifold $M$, and the canonical maps $\psi_{1}$ and $\psi_{2}$ of $M$ on $M_{1}$ and $M_{2}$, we get the double $M \sharp M$ of $(M, \partial M)$ as the quotient manifold of $M_{1} \amalg M_{2}$ via the equivalence relation: $\psi_{1}(x) \sim \psi_{2}(x)$ if and only if $x \in \partial M$. In other words, we define the doubled manifold as $(M \times\{1,-1\}) / \sim$, where the equivalence relation $\sim$ is defined as:

$$
(x, i) \sim(y, j) \text { if and only if }(x=y \text { and } i=j) \text { or }(x=y \in \partial M \text { and any } i, j)
$$

The two boundaries identified in this way yield a $(n-1)$-hypersurface named the equator of $M \sharp M$. The manifold $M \sharp M$ can be equipped with a structure of $C^{\infty}$ manifold in the following way:

let $p:(M \times\{1,-1\}) \rightarrow M \sharp M$ be the canonical surjection, $U \subset p(\partial M \times$ $\{-1\})=p(\partial M \times\{1\})$ an open neighbourhod in $M \sharp M$, and $N$ the $g$-unitary inward normal field of $\partial M$, then the local chart $\Phi$ is defined as:

$$
\Phi(t, x)=\left\{\begin{array}{ll}
p\left(\exp _{x}[t \cdot N(x)], 1\right) & \text { if } \quad t \geq 0 \\
p\left(\exp _{x}[-t \cdot N(x)],-1\right) & \text { if } t<0
\end{array} .\right.
$$

If $\varepsilon \leq \operatorname{inj}_{M}\left(\operatorname{inj}_{M}\right.$ the injectivity radius of $\left.M\right)$, the exponential normal map is a diffoeomorphism of $] 0, \varepsilon[\times \partial M$ on its image in $M$, and the changes of charts are $C^{\infty}$-maps. In what follows, we shall denote $p_{ \pm}(x)=p(x, \pm 1)$.

Let $j: M \rightarrow M \times\{1\}$ be the isometric immersion of $M$ in $M \times\{1\}$ and let $\Sigma: M \sharp M \rightarrow M \sharp M$ be the symmetry that swaps the two copies of $M$ in $M \sharp M$ with respect to the equator: $\Sigma(M \times\{1\})=(M \times\{-1\})$. The map $j$ induces the metric $g_{1}=j^{*}(g)$ on $M \times\{1\}$, (resp. the metric $g_{-1}=\Sigma^{*}\left(g_{1}\right)$ on $M \times\{-1\})$. The passage to the quotient with respect to the equivalent relation $\sim$ induces the metric $g \sharp g$ on $M \sharp M$.

Lemma 2.1. The metric $g \sharp g$, as defined above on $M \sharp M$ is $C^{0}$ but not $C^{1}$; moreover it is a $C^{0}$-limit of $C^{\infty}$-metrics $g_{k}$ defined on $M \sharp M$.

Proof. Let $i_{t}$ be the canonical injection of $\partial M$ in $]-\varepsilon, \varepsilon[\times M$ given by $i_{t}(x)=(t, x)$. The metric $g_{t}=i_{t}^{*}\left[\Phi^{*}(g \sharp g)\right]$, defined on $\partial M$, admits left and right derivatives which are generally different. Let $N$ be the inward normal 
ESTIMATES OF THE LAPLACIAN SPECTRUM AND BOUNDS OF

to the boundary and let $X$ be any tangent vector to $\partial M$. Recalling that $g_{ \pm 1}=\left(p_{ \pm 1}\right)^{*}(g \sharp g)$ and that $d\left[\left(p_{ \pm 1}^{-1}\right) \circ \Phi\right]\left(\frac{\partial}{\partial t}\right)= \pm N$, we get:

$$
\begin{aligned}
\left.\frac{d}{d t}\right|_{t= \pm 0} g_{t}(X, Y) & =\left.\left.\frac{\partial}{\partial t}\right|_{t= \pm 0}\left(\Phi^{*}(g \sharp g)\right)\right|_{(t, x)}(0, X)(0, Y) \\
& = \pm N \cdot g(X, Y) \\
& = \pm\left[g\left(\nabla_{X} N, Y\right)+g\left(X, \nabla_{Y} N\right)\right] \\
& = \pm 2 \operatorname{II}_{\partial M}(X, Y)
\end{aligned}
$$

being $\mathrm{II}_{\partial M}$ the second fundamental form of the equator (viewed as a hypersurface embedded in $M \sharp M)$. Save the exceptional case when II $=0$, there is a finite jump between the left and the right derivatives of $g$. The second assumption follows from a classic argument of density of $C^{\infty}$-functions converging in the $C^{0}$ topology toward a $C^{0}$-function using a sequence $g_{k} \rightarrow g \sharp g$ of $C^{\infty}$-metrics converging to the metric $g \sharp g$ in the $C^{0}$ topology.

\subsection{General inherited properties}

Let $(M, g)$ be a compact $C^{\infty}$ Riemannian manifold of dimension $n$, the metric $g$ and the Laplace operator are written as $g=\sum_{i, j} g_{i j} d x^{i} \otimes d x^{j}$ and $\Delta=\sqrt{\operatorname{det} g^{-1}} \cdot \frac{\partial}{\partial x^{i}}\left(\sqrt{\operatorname{det} g} \cdot g^{i j} \cdot \frac{\partial}{\partial x^{j}}\right)$ in a local system of coordinates $\left(x^{1}, x^{2}, \ldots, x^{n}\right)$. It is well known that the Laplacian is a self adjoint elliptic operator having a discrete sequence of positive eigenvalues going to infinity: $0 \leq \lambda_{0}<\lambda_{1} \leq \lambda_{2} \leq \cdots \leq \lambda_{i} \leq \cdots$. Moreover, each eigenspace $E\left(\lambda_{i}\right)$ has finite dimension, the direct sum of them is dense in $C^{\infty}(M)$ and the Hilbert space $L^{2}\left(M, d v_{g}\right)\left(d v_{g}\right.$ is the Riemannian measure on $\left.M\right)$ has a Hilbertian base of eigenfunctions.

Classic Lemma 2.2. For $C^{0}$-metrics on $(M \sharp M, g \sharp g)$ the spectrum of the Laplacian coincides with the critical values of the functional

$$
u \mapsto R(u)=\frac{\int_{M \sharp M}|d u|_{g \sharp g}^{2} d v_{g \sharp g}}{\int_{M \sharp M} u^{2} d v_{g \sharp g}}
$$

defined on $\mathcal{H}_{0}=H_{1}(M, g) \backslash\{0\}$. The critical points are calculated using the min-max principle or the max-min principle, i.e.

$$
\begin{aligned}
\lambda_{i}(M \sharp M, g \sharp g) & =\inf _{\mathcal{E}_{i+1}} \max _{u \in \mathcal{E} \backslash\{0\}} \frac{\int_{M \sharp M}|d u|_{g \sharp \sharp}^{2} d v_{g \sharp g}}{\int_{M \sharp M} u^{2} d v_{g \sharp g}} \\
& =\sup _{\mathcal{E}_{i}} \inf _{u \in \mathcal{E}_{i}^{\perp} \backslash\{0\}} \frac{\int_{M \sharp M}|d u|_{g \sharp g}^{2} d v_{g \sharp g}}{\int_{M \sharp M} u^{2} d v_{g \sharp g}}
\end{aligned}
$$

being $\mathcal{E}_{i} \subset \mathcal{H}_{0}$ any vectorial subspace of dimension $i$ in $\mathcal{H}_{0}$. 
ESTIMATES OF THE LAPLACIAN SPECTRUM AND BOUNDS OF

TOPOLOGICAL INVARIANTS FOR RIEMANNIAN MANIFOLDS WITH

BOUNDARY.

Proof. See [6], Theorem 4.62.

Lemma 2.3. With the assumptions of the Classic Lemma 2.2 we get:

(i) $\operatorname{diam}(M \sharp M, g \sharp g)=\lim _{k \rightarrow+\infty} \operatorname{diam}\left(M \sharp M, g_{k}\right)$;

(ii) $\operatorname{Vol}(M \sharp M, g \sharp g)=\lim _{k \rightarrow+\infty} \operatorname{Vol}\left(M \sharp M, g_{k}\right)$;

(iii) $\lambda_{i}(M \sharp M, g \sharp g)=\lim _{k \rightarrow+\infty} \lambda_{i}\left(M \sharp M, g_{k}\right)$;

(iv) $h_{(M \sharp M, g \sharp g)}(\beta)=\lim _{k \rightarrow+\infty} h_{\left(M \sharp M, g_{k}\right)}(\beta)$ ( $h$ is here the isoperimetric profile of the manifold $(M \sharp M, g \sharp g))$.

Proof of (i) and (ii). These proofs are trivial because the volume and the diameter are both $C^{0}$ invariants.

Proof of (iii). Let $\left\{g_{k}\right\}_{k \in \mathbb{N}}$ be a sequence of metrics converging to the metric $g$ in the $C^{0}$-topology and let $C_{1}<C_{2}$ be two strictly positive constants such that $C_{1} g \leq g_{k} \leq C_{2} g$. For any $f \in H^{2}(M \sharp M)$ we have:

$$
\begin{gathered}
C_{1}^{\frac{n}{2}} \int_{M \sharp M} f^{2} d v_{g} \leq \int_{M \sharp M} f^{2} d v_{g_{k}} \leq C_{2}^{\frac{n}{2}} \int_{M \sharp M} f^{2} d v_{g} ; \\
\frac{C_{1}^{\frac{n}{2}}}{C_{2}} \int_{M \sharp M}|d f|^{2} d v_{g} \leq \int_{M \sharp M}|d f|^{2} d v_{g_{k}} \leq \frac{C_{2}^{\frac{n}{2}}}{C_{1}} \int_{M \sharp M}|d f|^{2} d v_{g} ;
\end{gathered}
$$

and

$$
\frac{C_{1}^{\frac{n}{2}}}{C_{2}^{\frac{n}{2}+1}} R_{g}(f) \leq R_{g_{k}}(f) \leq \frac{C_{2}^{\frac{n}{2}}}{C_{1}^{\frac{n}{2}+1}} R_{g}(f) .
$$

The use of the min-max principle yields

$$
\frac{C_{1}^{\frac{n}{2}}}{C_{2}^{\frac{n}{2}+1}} \lambda_{i}(g) \leq \lambda_{i}\left(g_{k}\right) \leq \frac{C_{2}^{\frac{n}{2}}}{C_{1}^{\frac{n}{2}+1}} \lambda_{i}(g) .
$$

We conclude that keeping $\frac{1}{C_{2}}=C_{1}=C_{k}$ such that $\lim _{k \rightarrow+\infty} C_{k}=1$ and making the $\lim _{k \rightarrow+\infty} \lambda_{i}\left(g_{k}\right)$.

Proof of (iv). Let $\Omega \subset M \sharp M$ be a piecewise regular boundary subset of $M \sharp M$ and let $\varepsilon$ be a strictly positive constant, we define

$$
\operatorname{Vol}_{g \sharp g}(\partial \Omega)=\lim _{\varepsilon \rightarrow 0^{+}} \frac{1}{\varepsilon} \operatorname{Vol}_{g \sharp g}\left\{x \in \Omega \text { s.t. } d_{g \sharp g}(x, \partial \Omega)<\varepsilon\right\} .
$$


ESTIMATES OF THE LAPLACIAN SPECTRUM AND BOUNDS OF

Let $\Omega_{\varepsilon}$ be the subset of $M \sharp M$ as $\Omega_{\varepsilon}=\left\{x \in \Omega\right.$ s.t. $\left.d_{g \sharp g}(x, \partial \Omega)<\varepsilon\right\}$. If any sequence of $\varepsilon_{k} \rightarrow 0^{+}$is such that $\left(1-\varepsilon_{k}\right)^{2} g_{k} \leq g \sharp g \leq\left(1+\varepsilon_{k}\right)^{2} g_{k}$, we get

$$
\left\{x \in \Omega \text { s.t. } d_{g_{k}}(x, \partial \Omega)<\frac{\varepsilon}{1+\varepsilon_{k}}\right\} \subset \Omega_{\varepsilon} \subset\left\{x \in \Omega \text { s.t. } d_{g_{k}}(x, \partial \Omega)<\frac{\varepsilon}{1-\varepsilon_{k}}\right\}
$$

For any integer $k$ we deduce that,

$$
\lim _{\varepsilon \rightarrow 0^{+}} \frac{\left(1-\varepsilon_{k}\right)^{n+1}}{\varepsilon} \operatorname{Vol}_{g \sharp g}\left(\Omega_{\varepsilon}\right) \leq \operatorname{Vol}_{g_{k}}(\partial \Omega) \leq \lim _{\varepsilon \rightarrow 0^{+}} \frac{\left(1+\varepsilon_{k}\right)^{n+1}}{\varepsilon} \operatorname{Vol}_{g \sharp g}\left(\Omega_{\varepsilon}\right),
$$

then $\operatorname{Vol}_{g \sharp g}(\partial \Omega)=\lim _{k \rightarrow+\infty} \operatorname{Vol}_{g_{k}}(\partial \Omega)$.

Definition 2.4. Let $N$ the inward unit normal to the boundary $\partial M$; a function $u \in C^{\infty}(M, \partial M, g)$ solves

- the Dirichlet problem when

$$
\left\{\begin{array}{l}
\Delta u=0 \\
\left.u\right|_{\partial M}=0
\end{array}\right.
$$

- the Neumann problem when

$$
\left\{\begin{array}{l}
\Delta u=0 \\
\left.\frac{\partial u}{\partial N}\right|_{\partial M}=0
\end{array}\right.
$$

Classic Lemma 2.5. Let $(M, \partial M, g)$ be a Riemannian manifold with nonempty boundary and $(M \sharp M, g \sharp g)$ its double; then

(i) the spectrum of $(M \sharp M, g \sharp g)$ is the union of the Dirichlet and of the Neumann spectra of $(M, \partial M, g)$ :

$$
\left\{\lambda_{i}(M \sharp M, g \sharp g) \mid i \in \mathbb{N}\right\}=\left\{\lambda_{i}^{D}(M)_{i} \mid i \in \mathbb{N} \backslash\{0\}\right\} \cup\left\{\lambda_{i}^{N}(M)_{i} \mid i \in \mathbb{N}\right\},
$$

each eigenvalue has to be counted with its own multiplicity;

(ii) there exists a Hilbertian base of eigenfunctions such that the restriction to each copy of $M$ is an eigenfunction of the Dirichlet or Neumann problem. 
ESTIMATES OF THE LAPLACIAN SPECTRUM AND BOUNDS OF

Proof. Let $\Sigma: M \sharp M \rightarrow M \sharp M$ be the symmetry that swaps the two copies of $M$ with respect of the equator; for every $f \in C^{\infty}(M \sharp M)$ this symmetry acts on each eigenspace $E_{\lambda}$ of the Laplacian of $(M \sharp M, g \sharp g)$ in a stable manner because

$$
\triangle(f \circ \Sigma)=\triangle f \circ \Sigma \text {. }
$$

Since $\Sigma$ is an involution map, each eigenspace $E_{\lambda}$ breaks into a direct sum of two parts:

$$
E_{\lambda}^{+}=\left\{u \in E_{\lambda} \mid u \circ \Sigma=u\right\} \quad \text { and } \quad E_{\lambda}^{-}=\left\{u \in E_{\lambda} \mid u \circ \Sigma=-u\right\} .
$$

It is clear that each element of $E_{\lambda}^{+}$(resp. of $E_{\lambda}^{-}$) satisfies the Neumann condition $\left.\frac{\partial u}{\partial N}\right|_{\partial M}=0$ (resp. the Dirichlet condition $\left.u\right|_{\partial M}=0$ ) for $(M, g)$, thus the spectrum of $(M \sharp M, g \sharp g)$ is included in the union of the Dirichlet and Neumann spectra of $(M, \partial M, g)$, i.e.

$$
\left\{\lambda_{i}(M \sharp M, g \sharp g) \mid i \in \mathbb{N}\right\} \subseteq\left\{\lambda_{i}^{D}(M)_{i} \mid i \in \mathbb{N} \backslash\{0\}\right\} \cup\left\{\lambda_{i}^{N}(M)_{i} \mid i \in \mathbb{N}\right\} .
$$

On the other hand, each $u \in C^{\infty}(M)$ verifying the Neumann conditions $\triangle u=0$ and $\left.\frac{\partial u}{\partial N}\right|_{\partial M}=0$ (resp. the the Dirichlet conditions $\triangle u=0$ and $\left.\left.u\right|_{\partial M}=0\right)$ can be extended by symmetry to a function of $C^{1}(M \sharp M)$ class such that $\triangle u=\lambda u$. Thereby the inverse inclusion is

$$
\left\{\lambda_{i}^{D}(M)_{i} \mid i \in \mathbb{N} \backslash\{0\}\right\} \cup\left\{\lambda_{i}^{N}(M)_{i} \mid i \in \mathbb{N}\right\} \subseteq\left\{\lambda_{i}(M \sharp M, g \sharp g) \mid i \in \mathbb{N}\right\} .
$$

and equality follows from the double inclusion. This equality also proves the existence of a Hilbertian base of eigenfunctions. Moreover, the restriction of each element of this Hilbertian base to each copy of $M \sharp M$ is an eigenfunction for the Dirichlet or the Neumann problem.

Example (Classic) 2.6. The sphere $\left(\mathbb{S}^{1}\right.$, can $)$ is the double of the Riemannian manifold with boundary $[0, \pi]$.

- The Dirichlet spectrum of $[0, \pi]$ is $\Sigma^{D}=\left\{k^{2} \mid k \in \mathbb{N} \backslash\{0\}\right\}$, the multiplicity of each eigenvalue is 1 and the eigenspace associated to $k^{2}$ is given by $E_{k^{2}}^{[0, \pi]}=\operatorname{Span}(\sin k t)$; 
ESTIMATES OF THE LAPLACIAN SPECTRUM AND BOUNDS OF TOPOLOGICAL INVARIANTS FOR RIEMANNIAN MANIFOLDS WITH BOUNDARY.

- the Neumann spectrum of $[0, \pi]$ is $\Sigma^{N}=\left\{k^{2} \mid k \in \mathbb{N}\right\}$, the multiplicity of each eigenvalue is 1 and the eigenspace associated to $k^{2}$ is given by $E_{k^{2}}^{[0, \pi]}=\operatorname{Span}(\cos k t)$;

- the spectrum of $\left(\mathbb{S}^{1}\right.$, can $)$ is thus $\Sigma^{\mathbb{S}^{1}}=\left\{k^{2} \mid k \in \mathbb{N}\right\}$, the zero-eigenvalue has multiplicity 1 and each strictly positive eigenvalue has multiplicity equal to 2 ; counting each eigenvalue with its multiplicity, for $k \geq 1$ we get $E_{k^{2}}^{\mathbb{S}^{1}}=\operatorname{Span}(\sin k t, \cos k t)$, or equally $\Sigma^{\mathbb{S}^{1}}=\Sigma^{D} \cup \Sigma^{N}$.

Classic Lemma 2.7. Let $(M, \partial M)$ be a Riemannian manifold with nonempty boundary and $M \sharp M$ its double, we have:

(i) $\operatorname{dim} H^{i}(M \sharp M)=\operatorname{dim} H^{i}(M, \partial M)+\operatorname{dim} H^{i}(M)$, where $H^{i}(M, \partial M)$ is the $i$-th group of relative cohomology of $(M, \partial M)$ and $H^{i}(M)$ is the $i-t h$ group of cohomology of $(M, \partial M)$;

(ii) if the dimension of $M$ is 2 and the boundary is connected, then the genus $\gamma$ of the double is such that $\gamma(M \sharp M)=2 \gamma(M)$.

Proof. See [9], (2011).

\section{Estimates depending on the passage to the limit for a sequence of $C^{\infty}$-metrics}

Remark 3.1. In what follows we shall denote by $\lambda_{1}^{D}(M, g)$ (resp. $\left.\lambda_{1}^{N}(M, g)\right)$ the first eigenvalue of the Dirichlet spectrum (resp. the first non-zero eigenvalue of the Neumann spectrum).

The first estimation, valid for 2-dimensional Riemannian manifolds only, depends on its genus $\gamma$; P. Yang and S. T. Yau - in 1980 - proved the following

Theorem 3.2. (Yang- Yau, [14]) Let $g$ be any metric on a compact surface $(M, g)$ of genus $\gamma$, then there exists an explicit function $C$ depending on the genus $\gamma$ only such that

$$
\lambda_{1}(g) \cdot \operatorname{Vol}(g) \leq C(\gamma)
$$

The passage to the limit of the metric gets the following extension immediately:

Theorem 3.3. Let $(M, \partial M, g)$ be a two-dimensional compact Riemannian manifold of genus $\gamma$ and with non-empty and connected boundary, then for any metric $g$ on $M$ one gets

$$
\min \left[\lambda_{1}^{N}(M, g), \lambda_{1}^{D}(M, g)\right] \cdot \operatorname{Vol}_{g}(M, g) \leq \frac{1}{2} C(2 \gamma)
$$


ESTIMATES OF THE LAPLACIAN SPECTRUM AND BOUNDS OF TOPOLOGICAL INVARIANTS FOR RIEMANNIAN MANIFOLDS WITH BOUNDARY.

when the constant $C$, which is the same of Theorem 3.2, depends on the genus $\gamma$ only.

Proof. From Lemma 2.7 (ii) we have $\gamma(M \sharp M)=2 \gamma(M)$; moreover, let $g_{k}$ be a sequence of $C^{\infty}$ metrics on $M \sharp M$ converging to the metric $g \sharp g$ in the $C^{0}$-topology. The Theorem 3.2 gets

$$
\lambda_{1}\left(M \sharp M, g_{k}\right) \cdot \operatorname{Vol}\left(M \sharp M, g_{k}\right) \leq C(2 \gamma) .
$$

This inequality is still valid when $g_{k}$ is replaced by $g \sharp g$ because, for the Lemma 2.3, both $\lambda_{1}\left(g_{k}\right)$ and $\operatorname{Vol}\left(g_{k}\right)$ go to the limit:

$$
\begin{aligned}
& C(2 \gamma) \geq \lambda_{1}(M \sharp M, g \sharp g) \cdot \operatorname{Vol}(M \sharp M, g \sharp g) \\
= & 2 \min \left[\lambda_{1}^{D}(M, g), \lambda_{1}^{N}(M, g)\right] \cdot \operatorname{Vol}_{g}(M, g)
\end{aligned}
$$

If the Euler characteristic of $(M, \partial M)$ is strictly positive, Hersch proved in 1970 - the following

Theorem 3.4. (Hersch, [8]) Let g (resp. can) be any Riemannian metric (resp. the canonical metric) on $\mathbb{S}^{2}$ such that $\operatorname{Vol}(g)=\operatorname{Vol}($ can $)$, if $0=\lambda_{0}(g)<$ $\lambda_{1}(g) \leq \ldots \leq \lambda_{i}(g) \leq \ldots$ is the sequence of eigenvalues of the Laplacian of $\left(\mathbb{S}^{2}, g\right)$, each one counted with its own multiplicity, then

$$
\frac{1}{\lambda_{1}(g)}+\frac{1}{\lambda_{2}(g)}+\frac{1}{\lambda_{3}(g)} \geq \frac{1}{\lambda_{1}(\text { can })}+\frac{1}{\lambda_{2}(\text { can })}+\frac{1}{\lambda_{3}(\text { can })}
$$

the equality occurs if and only if the sphere is endowed with the canonical metric.

Moreover, recalling that for the canonical sphere $\left(\mathbb{S}^{2}\right.$, can $)$ the first non-zero eigenvalues are equal, $\lambda_{1}($ can $)=\lambda_{2}($ can $)=\lambda_{3}($ can $)$, the following corollary is a direct consequence.

Corollary 3.5. With the same assumptions of Theorem 3.4 we have

$$
\lambda_{1}(g) \operatorname{Vol}(g) \leq \lambda_{1}(\text { can }) \operatorname{Vol}(\text { can })
$$

Also in this case it is possible to obtain an extension of the Hersch Theorem for compact surfaces with non-empty boundary and diffeomorphic to a 2dimensional disc $\left(B^{2}, \partial B^{2}\right)$, via a simple passage to the limit of the metric; we get the following optimal result: 
ESTIMATES OF THE LAPLACIAN SPECTRUM AND BOUNDS OF TOPOLOGICAL INVARIANTS FOR RIEMANNIAN MANIFOLDS WITH BOUNDARY.

Theorem 3.6. Let $(M, \partial M)$ be a compact Riemannian surface with nonempty boundary, diffeomporphic to the disk $\left(B^{2}, \partial B^{2}\right)$, if $g$ is any metric such that $\operatorname{Vol}(g)=\frac{1}{2} \mathrm{Vol}\left(\mathbb{S}^{2}\right.$, can $)$ and if $\lambda_{i}(g), i=1,2,3$, are the smallest eigenvalues appearing in the union of the Dirichlet and Neumann spectra of $(M, \partial M)$, each one with its own multiplicity, and if $\lambda_{i}($ can $), i=1,2,3$ are the three smallest eigenvalues of $\left(\mathbb{S}^{2}\right.$, can $)$, then

$$
\frac{1}{\lambda_{1}(g)}+\frac{1}{\lambda_{2}(g)}+\frac{1}{\lambda_{3}(g)} \geq \frac{1}{\lambda_{1}(\text { can })}+\frac{1}{\lambda_{2}(\text { can })}+\frac{1}{\lambda_{3}(\text { can })}=\frac{3}{2} .
$$

Proof. Assuming that $(M, \partial M)$ is diffeormorphic to $\left(B^{2}, \partial B^{2}\right)$ implies that $M \sharp M$ is diffeomorphic to $\mathbb{S}^{2}$. Let $g_{k}$ be a sequence of $C^{\infty}$-metrics on $M \sharp M$ converging to $g \sharp g$ in the $C^{0}$-topology; according to the Hersch Theorem 3.4 it follows that

$\frac{\operatorname{Vol}\left(\mathbb{S}^{2}, \text { can }\right)}{\operatorname{Vol}\left(g_{k}\right)} \cdot\left[\frac{1}{\lambda_{1}\left(g_{k}\right)}+\frac{1}{\lambda_{2}\left(g_{k}\right)}+\frac{1}{\lambda_{3}\left(g_{k}\right)}\right] \geq \frac{1}{\lambda_{1}(\text { can })}+\frac{1}{\lambda_{2}(\text { can })}+\frac{1}{\lambda_{3}(\text { can })}$.

We proved in the Lemma 2.3 that $\lambda_{i}\left(g_{k}\right)$ and $\operatorname{Vol}\left(g_{k}\right)$ go to the limit $C^{0}$, pushing $k \rightarrow+\infty$ and applying this Lemma we get

$\frac{\operatorname{Vol}\left(\mathbb{S}^{2}, c a n\right)}{\operatorname{Vol}(g \sharp g)} \cdot\left[\frac{1}{\lambda_{1}(g \sharp g)}+\frac{1}{\lambda_{2}(g \sharp g)}+\frac{1}{\lambda_{3}(g \sharp g)}\right] \geq \frac{1}{\lambda_{1}(\text { can })}+\frac{1}{\lambda_{2}(\text { can })}+\frac{1}{\lambda_{3}(\text { can })} ;$

the use of the Classic Lemma 2.5 and the normalization $\operatorname{Vol}(g \sharp g)=\operatorname{Vol}($ can $)$ conclude the proof.

\section{Results depending on the lower bound of Ricci curva- ture}

Estimates from below the first eigenvalue have been obtained from several authors for Riemannian manifolds with empty or non-empty boundaries with Dirichlet or Neumann data on the boundary, under the the strong and unavoidable condition of the finite lower bound of Ricci curvature. It is clear that, gluing two isometric copies of the same Riemmanian manifold with nonempty boundary along their common boundary, the Ricci curvature can reach negative values that are still high in a neighbourhod of the junction, it just depends on the shape of the boundary. We give here the following definition:

Definition 4.1. A Riemmanian manifold with non-empty boundary $(M, \partial M, g)$ is said to be with convex boundary if the second fundamental form of the boundary $I I_{\partial M}$ is negative definite with respect to the inward normal $N$. 
ESTIMATES OF THE LAPLACIAN SPECTRUM AND BOUNDS OF TOPOLOGICAL INVARIANTS FOR RIEMANNIAN MANIFOLDS WITH BOUNDARY.

The lower bound of the first eigenvalue for Riemannian manifolds with empty and non-empty boundary with Dirichlet data was obtained in 1980 by $\mathrm{Li}$ and Yau [10]. These results are valid for both convex and non-convex boundaries, the inequality is, however, stronger in the convex case. In 1986 Meyer [11] obtained an analogous estimation for the first eigenvalue of the Laplacian on a Riemannian manifold with non-empty boundary with Neumann data, in the case of both convex and non-convex boundary. In his work he pointed out the blow up of the estimate when the injectivity radius of the boundary vanishes if the boundary is non-convex.

The new metric obtained in the doubled manifold ( $M \sharp M, g \sharp g$ ) has to obey the necessary Ricci curvature bounded from above condition and reflect the different behavior of convex or non-convex boundary. Thanks to the Main Theorems A and B proved in the last Section, we obtain the new $C^{2}$-metric on the doubled manifold such that

- if the boundary is convex, the control from below the curvature immediately follows (Main Theorem A);

- if the boundary is not-convex, the control of the curvature is still obtained, but takes into account the geometry of the boundary via its curvature and injectivity radius, which is consistent with the Meyer's results (Main Theorm B).

Thanks to this regularization, it is possible to extend the following results concerning $n$-dimen-sional compact Riemannian manifolds to compact Riemannian manifolds with non-empty boundary .

Theorem 4.2. (Payne and Weinberger (1957), [12]; Li and Yau (1980), [10]; Meyer (1986), [11]; Gallot (1983), [3], [4] and [5]) Let $(M, g)$ be a $C^{\infty}$ compact Riemannian manifold of dimenision $n$, and $\delta$ and $D$ two strictly positive constants such that Ricci $g \geq-(n-1) \delta^{2} g$ and $\operatorname{diam}(M, g) \leq D$, then there exists a constant $C$, that depends only on the product $\delta \cdot D$, such that the first non-zero eigenvalue of Laplacian is greater then this constant:

$$
\lambda_{1}(g) \cdot \operatorname{diam}^{2}(M, g) \geq C(\delta \cdot D) .
$$

Theorem 4.3. (Gromov (1981), [7]; Gallot (1983) [4]) Let $(M, g)$ be a compact $n$-dimensional Riemannian manifold, and $\delta$ and $D$ two positive numbers such that Ricci $_{g} \geq-\delta^{2}(n-1) g$ and $\operatorname{diam}_{g}(M) \leq D$, then there exists a constant $C$, that depends on the product $\delta \cdot D$ and on the dimension $n$, such that 
ESTIMATES OF THE LAPLACIAN SPECTRUM AND BOUNDS OF TOPOLOGICAL INVARIANTS FOR RIEMANNIAN MANIFOLDS WITH BOUNDARY.

$$
\operatorname{dim} H^{1}(M) \leq n \cdot \exp (n \delta \cdot D C(\delta \cdot D))
$$

Remark 4.4. In what follows we shall assume that the manifold $M \sharp M$ is already equipped with the suitable metric $\tilde{g} \sharp \tilde{g}$.

\subsection{Riemannian manifolds with convex boundary}

All the above presented results are also extended here to $n$-dimensional Riemannian manifolds with non-empty and convex boundaries.

Theorem 4.5. Let $(M, \partial M, g)$ be a n-dimensional Riemannian manifold with convex boundary and $\delta$ and $D$ two strictly positive real numbers such that Ricci $_{g} \geq-(n-1) \delta^{2} \cdot g$ and $\operatorname{diam}(M) \leq D$, then there exists a constant $C$ that depends only on the product $\delta D$ and on the dimension $n$ such that

$$
\begin{gathered}
\lambda_{1}^{N}(M, \partial M, g) \cdot \operatorname{diam}^{2}(M, \partial M, g) \geq C \\
\lambda_{1}^{D}(M, \partial M, g) \cdot \operatorname{diam}^{2}(M, \partial M, g) \geq C .
\end{gathered}
$$

Proof. We consider the double $M \sharp M$; from the Main Theorem A there exists a sequence of $C^{\infty}$ metrics $\left\{g_{k}\right\}_{k \in \mathbb{N}}$ converging in the $C^{0}$-topology to $g \sharp g$, such that $\min \left(\sigma_{g_{k}}\right) \geq \min \left(\sigma_{g}\right)$ for every $k \in \mathbb{N}$; this implies Ricci $i_{g_{k}} \geq$ $-(n-1) \delta^{2} \cdot g_{k}$. The use of the Theorem 4.2 to the manifold $\left(M \sharp M, g_{k}\right)$ gets

$$
\lambda_{1}\left(M \sharp M, g_{k}\right) \cdot \operatorname{diam}^{2}\left(M \sharp M, g_{k}\right) \geq C\left(\delta \cdot D_{g_{k}}\right)
$$

Pushing $k$ to infinity we have

$$
\lambda_{1}(M \sharp M, g \sharp g) \cdot \operatorname{diam}^{2}(M \sharp M, g \sharp g) \geq C\left(\delta \cdot D_{g \sharp g}\right)
$$

from the fact that $\operatorname{diam}_{g \sharp g} \leq 2$ diam and for the Lemma 2.3 we get

$$
\begin{gathered}
\lambda_{1}^{N}(M, \partial M, g) \cdot \operatorname{diam}^{2}(M, \partial M, g) \geq C \\
\lambda_{1}^{D}(M, \partial M, g) \cdot \operatorname{diam}^{2}(M, \partial M, g) \geq C .
\end{gathered}
$$

which concludes.

The following Proposition extends the results of the Theorem 4.3 to compact Riemannian manifolds with non empty and convex boundary: 
ESTIMATES OF THE LAPLACIAN SPECTRUM AND BOUNDS OF TOPOLOGICAL INVARIANTS FOR RIEMANNIAN MANIFOLDS WITH BOUNDARY.

Theorem 4.6. From the assumptions made in the Proposition 4.5 it also follows that

$$
\operatorname{dim} H^{1}(M, \mathbb{R})(M)+\operatorname{dim} H^{1}(M, \partial M) \leq n \cdot \exp (n \delta D \cdot C(\delta D))
$$

where $C$, which is the same as in Theorem 4.3, is a constant that depends on the product $\delta D$ only.

Proof. From the Main Theorem A on $M \sharp M$ there exists a sequence of $C^{\infty}$-metrics $g_{k}$ converging in the $C^{0}$-topology to the $C^{\infty}$-metric $g \sharp g$, such that $\min \sigma_{g_{k}} \geq \sigma_{g}$ for all $k$, then there exists a sequence of $C^{\infty}$-metrics $g_{k}$, such that Ricci $_{g_{k}} \geq-(n-1) \delta^{2} g$. From the Classic Lemma 2.7 and the Theorem 4.3 we deduce $\operatorname{dim} H^{1}(M, \mathbb{R})(M)+\operatorname{dim} H^{1}(M, \partial M)=\operatorname{dim} H^{1}(M \sharp M) \leq$ $n \cdot \exp (n \delta D \cdot C(\delta D))$ where $C$ is the constant of Theorem 4.3.

\subsection{Riemannian manifolds with non-convex boundary}

Similarly, we extend here all estimates, for which a control from above the curvature is necessary, to Riemannian manifolds with non-empty and nonconvex boundaries.

Theorem 4.7. Let $n>2$ be an integer and let $D$ and a be two strictly positive real numbers; let $\eta$ and $k$ be two real numbers. For every compact connected $n$-dimensional Riemannian manifold $(M, \partial M, g)$ whose diameter is bounded from above by $D$ and the sectional curvature is bounded from below by $-k^{2}$, and has a non-empty boundary $\partial M$ whose injectivity radius is bounded from below from a, and the main curvature is bounded from above by $\eta$, i.e.

$$
\operatorname{diam}(M) \leq D, \quad \sigma_{g} \geq-k^{2}, \quad \operatorname{inj}_{\partial M} \geq a, \quad \text { and } \quad h \leq \eta ;
$$

then there exists a constant $C^{\prime}$, that depends on $n, D, k, a$ and $\eta$, such that

$$
\begin{gathered}
\lambda_{1}^{D}(M, g) \cdot \operatorname{diam}^{2}(M, g) \geq C^{\prime}(n, D, k, a, \eta) \\
\lambda_{1}^{N}(M, g) \cdot \operatorname{diam}^{2}(M, g) \geq C^{\prime}(n, D, k, a, \eta) .
\end{gathered}
$$

Proof. As in the Main Theorem B, on the manifold $M \sharp M$, the metric $\tilde{g} \sharp \tilde{g}$ is such that $\sigma_{\tilde{g}} \geq \frac{-4 k^{2}}{\tanh ^{2}\left(\frac{k a}{4}\right)}$. We can then apply Theorem 4.2 to the compact manifold with empty boundary $\left(M \sharp M, \tilde{g}_{\phi}\right)$, getting

$$
\lambda_{1}(M \sharp M, \tilde{g} \sharp \tilde{g}) \cdot \operatorname{diam}^{2}(M \sharp M, \tilde{g} \sharp \tilde{g}) \geq C\left(\frac{2 k \cdot D}{\tanh \left(\frac{k a}{4}\right)}\right)
$$


ESTIMATES OF THE LAPLACIAN SPECTRUM AND BOUNDS OF

TOPOLOGICAL INVARIANTS FOR RIEMANNIAN MANIFOLDS WITH

BOUNDARY.

and

$$
\begin{aligned}
& \lambda_{1}^{D}(M, g) \cdot \operatorname{diam}^{2}(M, g) \geq C\left(\frac{2 k \cdot D}{\tanh \left(\frac{k a}{4}\right)}\right) \\
& \lambda_{1}^{N}(M, g) \cdot \operatorname{diam}^{2}(M, g) \geq C\left(\frac{2 k \cdot D}{\tanh \left(\frac{k a}{4}\right)}\right) .
\end{aligned}
$$

where $C$ is the constant as in Theorem 4.2. For the sake of simplicity we now call

$u(a, k)=\left(\frac{\sinh \left(\frac{k a}{2}\right)}{\sinh \left(\frac{k a}{4}\right)}\right), \quad v(a, k, \eta)=\left[\cosh \left(\frac{k a}{4}\right)+\sup \left(1, \frac{\eta}{k}\right) \cdot \sinh \left(\frac{k a}{4}\right)\right]$

and applying the Main Theorem B we obtain

$$
u(a, k)^{-2} \cdot g \leq \tilde{g} \leq v(a, k, \eta)^{2 n-2} u(a, k)^{2 n-4} \cdot g
$$

From the results of Lemma 2.3 we get the following estimates:

$$
\operatorname{diam}(M, \tilde{g}) \leq \operatorname{diam}(M, g) \cdot v(a, k, \eta)^{n-1} \cdot u(a, k)^{n-2}
$$

and

$$
\lambda_{1}(\tilde{g}) \leq u(a, k)^{n^{2}-n} v(a, k, \eta)^{n^{2}-n+2} \cdot \lambda_{1}(g) .
$$

Putting the previous inequalities in (4.1) we obtain

$$
\begin{aligned}
& \lambda_{1}^{D}(M \sharp M, \tilde{g} \sharp \tilde{g}) \cdot \operatorname{diam}^{2}(M, g) \geq C^{\prime}(n, D, k, a, \eta) \\
& \lambda_{1}^{N}(M \sharp M, \tilde{g} \sharp \tilde{g}) \cdot \operatorname{diam}^{2}(M, g) \geq C^{\prime}(n, D, k, a, \eta)
\end{aligned}
$$

being $C^{\prime}(n, D, k, a, \eta)=\frac{1}{4} C\left(\frac{2 k \cdot D}{\tanh \left(\frac{k a}{4}\right)}\right) \cdot u(a, k)^{2-n^{2}-n} v(a, k, \eta)^{2-n^{2}-n}$.

The inequalities from 4.2 show the existence of the constant $C^{\prime}$ that depends on $n, D, k, a$ and $\eta$ as in the statement, and concurrently give a lower bound of the first non-zero eigenvalue of the Laplacian of the manifold $(M, \partial M, g)$.

Theorem 4.8. With the same assumptions of the Theorem 4.7, we have:

$$
\operatorname{dim} H^{1}(M)+\operatorname{dim} H^{1}(M, \partial M) \leq n \cdot \exp \left(n \cdot C^{\prime}(n, D, k, a, \eta)\right)
$$

where $C^{\prime}(n, D, k, a, \eta)$ is a constant that depends only on $n, D, k, a, \eta$. 
ESTIMATES OF THE LAPLACIAN SPECTRUM AND BOUNDS OF

Proof. The proof is the same as in Proposition 4.6, but, because of the regularization of the metric, in this case the constant $C^{\prime}$ is given by

$$
C^{\prime}(n, D, k, a, \eta)=C\left[\frac{4 k}{\tanh \left(\frac{a k}{4}\right)} \cdot D \cdot u(a, k)^{n-2} \cdot v(a, k, \eta)^{n-1}\right]
$$

where $C$ is the constant of Theorem 4.3.

\section{Concluding remarks}

Despite the fact that it is impossible to directly compare the results of Theorems 4.7 and 4.8 with the previous results of $\mathrm{Li}$ and $\mathrm{Yau}[10]$ and Meyer [11] due to the high complexity of the involved constants, we emphasize here that the above presented method of proof is however significantly different from the classical gradient methods used by previous authors. The method of the double manifold shows indeed that we can obtain eigenvalue estimates for the manifold with boundary from a related manifold without boundary, namely its double. The unitary approach of the double makes it possible to get the estimates for both Dirichlet or Neumann data on the boundary; the Li and Yau approach did indeed obtain estimates only for Dirichlet data, whilst Meyer obtained only for Neumann data. Moreover, using the doubled manifold techniques it is possible to get estimates of other quantities such as the isoperimetric profile or the heat kernel.

\section{Appendix: Proofs of the Main Theorems}

We present here how to get a $C^{\infty}$ metric on the doubled manifold $(M \sharp M, \tilde{g} \sharp \tilde{g})$ with a suitable control from below the sectional curvature with the strong assumption that the map $(M \sharp M, \tilde{g} \sharp \tilde{g}) \rightarrow(M \sharp M, g \sharp g)$ be a local isometry in a consistent neighbourhod of the equator.

\subsection{Comparison of the principal curvatures of hypersurfaces}

Lemma 6.1. Let $(M, \partial M, g)$ be a compact Riemannian manifold with nonempty boundary $\partial M$ and $\delta$ and $k$ two real numbers such that

$$
\text { Ricci }_{g} \geq-(n-1) \cdot \delta^{2} g \quad \text { and } \quad \sigma_{g} \geq-k^{2} .
$$

Let a (resp. $\eta)$ be the lower bound of the injectitvity radius of the boundary (resp. the upper bound of the mean curvature of the boundary). Let $H_{t}=$ 
ESTIMATES OF THE LAPLACIAN SPECTRUM AND BOUNDS OF

$\{x \in M$ s.t. $d(x, \partial M)=t\}$ be the hypersurface at $t$ distance form the boundary and let $h_{t}$ be its second fundamental form. Let $h_{\min }(t)$ and $h_{\max }(t)$ be the minimal and maximal eigenvalues of $h_{t}$, then, for every $\left.\varepsilon \in\right] 0, \frac{a}{2}[$ and in every point of $H_{t}$ we get:

(i) for every $t \in\left[0, \frac{a}{2}\right]$

$$
-(n-1) \cdot \frac{\delta}{\tanh \left(\frac{a \delta}{2}\right)} \leq \operatorname{trace}\left(h_{t}\right) \leq(n-1) \cdot \sup (1, \eta) ;
$$

(ii) for every $t \in\left[\varepsilon, \frac{a}{2}\right]$

$$
-(n-1) \cdot \frac{\delta}{\tanh \left(\frac{a \delta}{2}\right)} \leq \operatorname{trace}\left(h_{t}\right) \leq(n-1) \cdot \frac{\delta}{\tanh (\varepsilon \delta)}
$$

(iii) for every $t \in\left[\varepsilon, \frac{a}{2}\right]$

$$
-\frac{k}{\tanh \left(\frac{a k}{2}\right)} \leq h_{\min }(t) \leq h_{\max }(t) \leq \frac{k}{\tanh (\varepsilon k)} ;
$$

(iv) $-\frac{k}{\tanh \left(\frac{a k}{2}\right)} \leq h_{\min }(t) \leq$

$$
h_{\text {max }}(t) \leq(n-1) \cdot \sup (1, \eta)+(n-2) \cdot \frac{k}{\tanh \left(\frac{a k}{2}\right)} .
$$

Proof of (i). Left inequality: Let $c$ be the geodesic, normal to the boundary $\partial M$ in $c(0) \in \partial M$ and parametrized for the arc length. The geodesic disk $B_{t}$, centred in $c(a)$ and with the radius $(a-t)$ is tangent to $H_{t}$ at the point $c(t)$. The geodesic $c$ yields the minimal distance between $c(a)$ and $H_{t}$, thus the hypersurface $H_{t}$ is external to the disk $B_{t}$. The principal and the mean curvatures of $H_{t}$ take their lower bound in the principal and the mean curvatures of $\partial B_{t}$.

Let $\left.\Phi: \mathbb{S}^{n-1} \times\right] 0,+\infty[$ be the inverse of a normal chart at $c(a)$, let $\Phi^{*} v_{g}=\alpha(\mathbf{u}, t) d \mathbf{u} \cdot d t$ be the volume form $\left(\alpha(\mathbf{u}, t)=t^{n-1} J(\mathbf{u}, t), J(\mathbf{u}, t)\right.$ is the density of the volume form in normal coordinates) and let $b=\alpha^{\frac{1}{n-1}}$. From the Bishop Comparison Theorem we deduce:

$$
\begin{gathered}
\operatorname{trace}\left(\partial B_{t}\right)=-(n-1) \frac{b^{\prime}}{b} \geq-(n-1) \cdot \frac{\delta(\sinh \delta(a-t))^{\prime}}{\sinh \delta(a-t)}= \\
-(n-1) \cdot \frac{\delta}{\tanh \delta(a-t)} \geq-(n-1) \cdot \frac{\delta}{\tanh \frac{\delta a}{2}} .
\end{gathered}
$$


ESTIMATES OF THE LAPLACIAN SPECTRUM AND BOUNDS OF

TOPOLOGICAL INVARIANTS FOR RIEMANNIAN MANIFOLDS WITH

BOUNDARY.

Right inequality. Let $\vartheta(t)$ be the volume form at point $(x, t)=\exp _{x}(t \cdot N(x))$, $d v_{g}=\vartheta(t) d x d t$ and let $\tilde{\vartheta}(t)=\left[\cosh \delta t+\frac{\eta(x)}{\delta} \cdot \sinh \delta t\right]^{n-1}$ where $\eta(x)$ is the mean curvature of the boundary at the point $c(0)$. The Heinze-Kärcher Theorem gives:

$$
\operatorname{trace}\left(h_{t}\right)=\frac{\vartheta^{\prime}}{\vartheta} \leq \frac{\tilde{\vartheta}^{\prime}}{\tilde{\vartheta}}
$$

If $\frac{\eta}{\delta} \in[-1,1]$, we set $\frac{\eta}{\delta}=\frac{\sinh \delta R}{\cosh \delta R}$, and since tanh is an increasing function of its argument, we get

$$
\operatorname{trace}\left(h_{t}\right) \leq(n-1) \cdot \delta \cdot \frac{\sinh \delta(R+t)}{\cosh \delta(R+t)} \leq(n-1) \delta .
$$

On the other hand, using the same arguments as in the proof of the left inequaility, if $\frac{\eta}{\delta} \notin[-1,1]$, we get:

$$
\operatorname{trace}\left(h_{t}\right) \leq(n-1) \cdot \delta \cdot \frac{\cosh \delta(R+t)}{\sinh \delta(R+t)} \leq(n-1) \cdot \delta \cdot \frac{\cosh (\delta R)}{\sinh (\delta R)} \leq(n-1) \eta .
$$

Proof of (ii). Right inequality. The geodesic disk $B^{\prime}(t)$, centred at $c(0)$ and with radius $t$ is tangent to $H_{t}$ in the point $c(t)$, it lies within $H_{t}$. The Comparison Theorems of Bishop and Rauch give, for every $t \in\left[\varepsilon, \frac{a}{2}\right]$,

$$
\operatorname{trace}\left(h_{t}\right) \leq(n-1) \cdot \frac{\delta}{\tanh (\delta t)} \leq(n-1) \cdot \frac{\delta}{\tanh (\delta \varepsilon)} .
$$

Proof of (iii). Left inequality. Let $J(t)$ be a Jacobi field along the geodesic $c(t)$, starting from $c(a)$, and normal to the boundary $\partial M$ and let $J_{0}$ and $g_{0}$ be respectively the Jacobi field and the metric, corresponding to $J(t)$ and $g$, on a reference space of constant sectional curvature equal to $-k^{2}$. From the Rauch Comparison Theorem we get:

$$
\begin{gathered}
h_{t}(J(t), J(t))=-\frac{g\left(J^{\prime}(t), J(t)\right)}{g(J(t), J(t))} \geq-\frac{g_{0}\left(J_{0}^{\prime}(t), J_{0}(t)\right)}{g_{0}\left(J_{0}(t), J_{0}(t)\right)}= \\
-\frac{k}{\tanh [k(a-t)]} \geq-\frac{k}{\tanh \left(\frac{a k}{2}\right)} .
\end{gathered}
$$

The right inequality follows from the right inequality of (ii) remembering that, for every $t \in\left[\varepsilon, \frac{a}{2}\right]$

$$
h_{\max } \leq \frac{k}{\tanh (\varepsilon k)} .
$$


ESTIMATES OF THE LAPLACIAN SPECTRUM AND BOUNDS OF TOPOLOGICAL INVARIANTS FOR RIEMANNIAN MANIFOLDS WITH BOUNDARY.

Proof of (iv). Right inequality. It is enough to emphasize that $h_{\max }(t)+(n-$ 2) $h_{\min }(t) \leq \operatorname{trace}\left(h_{t}\right)$, the use of the lower bound of $\operatorname{trace}\left(h_{t}\right)$ and the lower bound of $h_{\min }(t)$ as obtained above completes the proof of this Lemma.

Lemma 6.2. Let $(M, \partial M, g)$ be a $n$-dimensional Riemannian manifold whose sectional curvature belongs to the interval $[-1,1]$, with non-empty boundary $\partial M$ whose injectivity radius is greater than a strictly positive real constant a. Let $H_{t}=\{x \in M$ s.t. $d(x, \partial M)=t\}$ be the hypersurface at $t$-distance from the boundary $(t<a)$ and let $h_{t}$ be its second fundamental form (with respect to the inward normal). Running $x$ all over $H_{t}$, if

- $h_{\max }(t)$ is the maximum among the biggest eigenvalues of $h_{t}$ and

- $h_{\min }(t)$ is the minimum among the lowest eigenvalues of $h_{t}$,

then, for every $t<a$ we get

$h_{\max }(t) \leq \frac{h_{\max }(0)+\tanh t}{1+h_{\max }(0) \cdot \tanh t} \quad$ and $\quad h_{\min }(t) \geq \frac{h_{\min }(0)-\tan t}{1+h_{\min }(0) \cdot \tan t}$

Proof. Let $x \in H_{t}$ be a point of the hypersurface $H_{t}$. Let $c$ be the normal geodesic to the boundary $\partial M$, passing in $x$ and parametrized for the arc length. Let $h_{\max }(t, x)$ be the biggest eigenvalue of $h_{t}$ in the point $x$. For any Jacobi field along $c$ and normal to it we have:

$$
h_{\text {max }}(t, x)=\sup \frac{\left.h_{t}\right|_{x}(V, V)}{g(V, V)} .
$$

Let $\widetilde{V}$ be the Jacobi field which achieves the sup (this sup is attained because the Jacobi fields along the geodesic $c$ form a linear space of finite dimension), then $h_{\max }(t, x)=\frac{g\left(D_{\dot{c}} \widetilde{V}, \widetilde{V}\right)}{g(\widetilde{V}, \widetilde{V})}$. Taking $\widetilde{V}$ so that $\|\widetilde{V}(0)\|=1$, we have $\left\|\widetilde{V}^{\prime}(0)\right\|=\left\|D_{\dot{c}} \widetilde{V}(0)\right\|=\|\mathcal{L}(\widetilde{V})\|$ and $\mathcal{L}$ is here the Weingarten operator of $\partial M$ in the point $c(0)$; we set $\|\mathcal{L}(\widetilde{V})\|=\eta \leq h_{\text {max }}(0)$. To get such a comparison, we consider the corresponding geodesic $c_{0}$ and the Jacobi normal field $\widetilde{V}_{0}$ along the geodesic $c_{0}$ such that $\left\|\widetilde{V}_{0}\right\|=1$ and $\left\|\widetilde{V}_{0}^{\prime}\right\|=\eta$ on the hyperbolic space of the same dimension. This field is given by

$$
\widetilde{V}_{0}(t)=(\eta \cdot \sinh t+\cosh t) v(t)
$$

being $v(t)$ the parallel transport along $c_{0}$ of the vector $v(0)$ and such that $g_{0}(v(0), \dot{c}(0))=0$. From the Rauch Comparison Theorem, the condition $\sigma_{g} \geq$ -1 implies that 
ESTIMATES OF THE LAPLACIAN SPECTRUM AND BOUNDS OF

$$
\begin{aligned}
\frac{g\left(\widetilde{V}^{\prime}, \widetilde{V}\right)}{g(\widetilde{V}, \widetilde{V})} \leq \frac{g_{0}\left(\widetilde{V}_{0}^{\prime}, \widetilde{V}_{0}\right)}{g_{0}\left(\widetilde{V}_{0}, \widetilde{V}_{0}\right)} & =\frac{(\eta \cdot \cosh t+\sinh t)(\eta \cdot \sinh t+\cosh t)}{(\eta \cdot \sinh t+\cosh t)^{2}} \\
& =\frac{\eta \cdot \cosh t+\sinh t}{\eta \sinh t+\cosh t} \\
& =\frac{\eta+\tanh t}{1+\eta \cdot \tanh t}
\end{aligned}
$$

The function $\left(t, h_{\max }(0)\right) \mapsto \frac{\tanh t+h_{\max }(0)}{1+h_{\max }(0) \tanh t}$ is

- an increasing function of $h_{\max }(0)$;

- an increasing function of $t$ if $h_{\max }(0)<1$ and

- a decreasing function of $f$ if $h_{\max }(0)>1$.

The condition $h_{\max }(0) \geq \eta$ gets, for all $x \in H_{t}$

$$
h_{\max }(t, x) \leq \frac{\eta+\tanh t}{1+\eta \cdot \tanh t} \leq \frac{\tanh t+h_{\max }(0)}{1+h_{\max }(0) \cdot \tanh t}
$$

which proves the first inequality of (6.1).

Using as a reference space the sphere $\left(\mathbb{S}^{n-1}\right.$, can $)$ rather than the hyperbolic space $\left(\mathbb{H}^{n-1}\right.$, can $)$ we prove the second inequality of $(6.1)$; with the same notations, in $\left(\mathbb{S}^{n-1}\right.$, can $)$ the Jacobi field is given by $\widetilde{V}_{0}=(\eta \cdot \sin t+\cos t) v(t)$. We get

$$
h_{\min }(t, x) \geq \frac{\eta-\tan t}{1+\eta \cdot \tan t} ;
$$

the map $\left(t, h_{\min }(0)\right) \mapsto \frac{h_{\min }(0)-\tan t}{1+h_{\min }(0) \cdot \tan t}$ is a decreasing function of $t$ and an increasing function of $h_{\min }(0)$; we obtain, for all $x \in H_{t}$

$$
\frac{\eta-\tan t}{1+\eta \cdot \tan t} \geq \frac{h_{\min }(0)-\tan t}{1+h_{\min }(0) \cdot \tan t}
$$

proving the second inequality of (6.1).

\subsection{Comparison of metrics}

The aim of this section and of the next one is to get a $C^{\infty}-$ metric $\tilde{g}$ with a control from below the sectional curvature on the doubled manifold $(M \sharp M, g \sharp g)$. This new metric will allow us to prove the Main Theorems A and $\mathrm{B}$ at the end of the next section. 
ESTIMATES OF THE LAPLACIAN SPECTRUM AND BOUNDS OF TOPOLOGICAL INVARIANTS FOR RIEMANNIAN MANIFOLDS WITH BOUNDARY.

Let $a>0$ be the lower bound of the injectivity radius of the boundary $\partial M$ and let $N(x)$ be the inward unit normal at $x$ to $M$. We consider the exponential map $\Psi: \partial M \times\left[0, a\left[\rightarrow M, \Psi(x, t)=\exp _{x}(t \cdot N(x))\right.\right.$ and the canonical embedding $i_{t}$ of $\partial M$ in $\partial M \times[0, a[$ which identifies $\partial M$ with $\partial M \times\{t\}$ and we define $g_{t}=i_{t}^{*}\left[\Psi^{*} g\right]$. In the coordinates system stated by $\Psi$, an analogous theorem of the Gauss Lemma (see, e.g. [2], p. 69) makes it possible to write $g$ as $\left.\left(\Psi^{*} g\right)\right|_{(x, t)}=(d t)^{2} \oplus g_{t}$.

Let $l$ be a strictly positive constant such that $0<l<\frac{a}{2}$, and let $M_{\frac{a}{2}} \subset M$ be the cylinder $M_{\frac{a}{2}}=\left\{x \in M\right.$ s.t. $\left.d_{(x, \partial M)} \leq \frac{a}{2}\right\}$ endowed with the metric $g$ as defined above. Let lastly $\phi_{l}:\left[0,+\infty\left[\rightarrow\left[0,+\infty\left[\right.\right.\right.\right.$ be a $C^{\infty}$-function enjoying the following properties

p.1 $\phi_{l}(0)=l$;

p.2 $\phi_{l}^{(n)}(0)=0$ for all $n \geq 1$;

p.3 $\phi_{l}(t)=t$ for all $t \geq \frac{a}{2}$

p.4 $\phi_{l}^{\prime}(t)>0$ for all $t>0$;

p.5 $0 \leq \phi_{l}{ }^{\prime \prime} \leq \frac{1}{l}$

In the next developments, the constant $l$ will assume values suitable to the specific case.

The perturbed metric $\tilde{g}$ is given, in general, by

$$
\left.\tilde{g}\right|_{(t, x)}=\left\{\begin{array}{ccc}
\left.(d t)^{2} \oplus g_{\phi_{l}(t)}\right|_{x} & \text { on } & M_{\frac{a}{2}} \\
\left.g\right|_{(t, x)} & \text { on } & M \backslash M_{\frac{a}{2}}
\end{array}\right.
$$

With these assumptions we get $\left.\frac{d}{d t}\right|_{t=0} g_{\phi_{l}(t)}=\left.\phi_{l}^{\prime}(0) \cdot \frac{d}{d t}\right|_{t=\phi_{l}(0)} g_{t}=0$. The induced metric $\tilde{g}_{\phi_{l}} \sharp \tilde{g}_{\phi_{l}}$ is of class $C^{2}$ for reasons of symmetry; moreover, since all derivatives of $\phi_{l}$ vanish in $t=0$, the metric $\tilde{g}_{\phi_{l}} \sharp \tilde{g}_{\phi_{l}}$ is $C^{\infty}$.

Remark 6.3. The metric g yields a $C^{\infty}$-metric on $M \sharp M$ if and only if $\partial M$ is totally geodesic. Deriving in the vectorial space of symmetric 2-tensors on $\partial M$, we get $\left.\frac{d}{d t}\right|_{t=0} g_{t}=2 I I_{\partial M}$, being $I I_{\partial M}$ the second fundamental form of $\partial M$.

Proof. Let $(x, t) \in \partial M \times\left[0, a\left[\right.\right.$ be a point of $M$ and $\left(\frac{\partial}{\partial x_{1}}, \ldots, \frac{\partial}{\partial x_{n}}, \frac{\partial}{\partial t}\right)$ be the field given by a system of coordinates. The metric $g$ yields a $C^{\infty}$-metric on $M \sharp M$ if and only if $\left.\frac{\partial}{\partial t} \cdot g\left(\frac{\partial}{\partial x_{i}}, \frac{\partial}{\partial x_{j}}\right)\right|_{(x, 0)}=0$ for all $i$ and $j$. We have, in fact, 


$$
\begin{aligned}
\left.\frac{\partial}{\partial t} \cdot g\left(\frac{\partial}{\partial x_{i}}, \frac{\partial}{\partial x_{j}}\right)\right|_{(x, 0)}= & \left.g\left(D_{\frac{\partial}{\partial t}} \frac{\partial}{\partial x_{i}}, \frac{\partial}{\partial x_{j}}\right)\right|_{(x, 0)}+\left.g\left(\frac{\partial}{\partial x_{i}}, D_{\frac{\partial}{\partial t}} \frac{\partial}{\partial x_{j}}\right)\right|_{(x, 0)}= \\
& 2 \cdot \operatorname{II}(x)\left(\frac{\partial}{\partial x_{i}}, \frac{\partial}{\partial x_{j}}\right) .
\end{aligned}
$$

The goal is now is to give an estimation of the curvature of $\tilde{g}_{\phi_{l}}$; to do so, we compare the metric $\tilde{g}_{\phi_{l}}$ with a second metric $\bar{g}_{\phi_{l}}$ given by

$$
\bar{g}_{\phi_{l}}=\left\{\begin{array}{lll}
\frac{(d t)^{2}}{\left[\phi_{l}^{\prime}\left(\phi_{l}^{-1}\right)(t)\right]^{2}} \oplus g_{t} & \text { for } & \varepsilon \leq t \leq \frac{a}{2} \\
\bar{g}_{\phi_{l}}=\tilde{g}_{\phi}=g & \text { on } & M \backslash M_{\frac{a}{2}}
\end{array}\right.
$$

at the point $\Psi(x, t) \in M \backslash M_{\varepsilon}$.

In this context we take $l=\varepsilon \leq \frac{a}{2}, \phi_{\varepsilon}(0)=\varepsilon>0$. The metric $\bar{g}_{\phi_{\varepsilon}}$ is defined only on $M \backslash M_{\varepsilon}$ because $\phi$ admits the inverse only in [ $\varepsilon,+\infty[$.

Proposition 6.4. The manifold $\left(M, \tilde{g}_{\phi}\right)$ is isometric to $\left(M \backslash M_{\varepsilon}, \bar{g}\right)$.

Proof. Let $\Phi: M \rightarrow M \backslash M_{\varepsilon}$ be the map given by:

$$
\Phi(\Psi(x, t))=\left\{\begin{array}{ccc}
\Psi\left(x, \phi_{\varepsilon}(t)\right) & \text { on } & M_{\frac{a}{2}} \\
I d & \text { on } & M \backslash M_{\frac{a}{2}}
\end{array} ;\right.
$$

in the chart given by $\Psi$, i.e. $\left.\frac{\partial}{\partial t}\right|_{\Psi(x, t)}=\left.d \Psi\right|_{(x, t)}[(0,1)]$, we denote by $\frac{\partial}{\partial t}$ the derivation with respect to $t$, which gives us

$$
\left.d \Phi\right|_{\Psi(x, t)}\left(\frac{\partial}{\partial t}\right)=\left.\phi_{\varepsilon}^{\prime}(t) \cdot \frac{\partial}{\partial t}\right|_{\Psi(x, \phi(t))}
$$

and

$$
\begin{gathered}
\left.\left(\Phi^{*} \cdot \bar{g}_{\phi_{\varepsilon}}\right)\right|_{\Psi(x, t)}\left(\frac{\partial}{\partial t}, \frac{\partial}{\partial t}\right)=\left.\bar{g}_{\phi_{\varepsilon}}\right|_{\Psi\left(x, \phi_{\varepsilon}(t)\right)}\left(\phi_{\varepsilon}^{\prime}(t) \cdot \frac{\partial}{\partial t}, \phi_{\varepsilon}^{\prime}(t) \cdot \frac{\partial}{\partial t}\right) \\
=\frac{1}{\phi_{\varepsilon}\left(\phi_{\varepsilon}^{-1}(\phi(t))\right)^{2}} \cdot \phi_{\varepsilon}^{\prime}(t)^{2}=1=\left.\tilde{g}_{\phi}\right|_{(x, t)}\left(\frac{\partial}{\partial t}, \frac{\partial}{\partial t}\right) .
\end{gathered}
$$

Moreover, let $Y$ be a vectorial field tangent to $\partial M:\left.Y\right|_{\Phi(x, t)}=\left.d \Psi\right|_{(x, t)}(\mathbf{v}, 0)$, $(\mathbf{v}$ is a tangent vector to $\partial M)$. We have $\Phi_{*} Y=Y$ and $\left[\Phi^{*} \cdot \bar{g}_{\phi_{\varepsilon}}\right](Y, Y)=$ 
ESTIMATES OF THE LAPLACIAN SPECTRUM AND BOUNDS OF TOPOLOGICAL INVARIANTS FOR RIEMANNIAN MANIFOLDS WITH BOUNDARY.

$\tilde{g}_{\phi_{\varepsilon}}(Y, Y)$. The metrics $\Phi^{*} \bar{g}$ and $\tilde{g}_{\phi_{\varepsilon}}$ assume the same values on the $\left(\frac{\partial}{\partial t}, Y\right)$ couple, because $\frac{\partial}{\partial t}$ and $Y$ are orthogonal both for the metric $\tilde{g}_{\phi_{\varepsilon}}$ and $\bar{g}_{\phi_{\varepsilon}}$ and the map $\Phi_{*}$ preserves the orthogonality.

\subsection{Comparison and control of the curvatures}

In what follow we suppose that all geodesics are parametrized for the arc length.

Lemma 6.5. Let $a$ and $\varepsilon$ be two strictly positive constants such that $\operatorname{inj}_{\partial M} \geq$ $a$ and $0<\varepsilon<\frac{a}{2}$; let $q:\left[\varepsilon, \frac{a}{2}\right] \rightarrow \mathbb{R}^{+}$be $a C^{1}$ strictly positive function and

$$
g=d t^{2} \oplus g_{t} \quad \text { and } \quad \bar{g}=\frac{1}{q^{2}(t)} d t^{2} \oplus g_{t}
$$

two different metrics drawn in $M_{\frac{a}{2}} \backslash M_{\varepsilon}$ by the coordinate frame equipped by $\Psi$. If $R$ and $\bar{R}$ are the corresponding Riemann curvature tensors, then, for every vector $X, Y, Z, U, V$ tangent to the hypersurface $H_{t}$ at $t$-distance form the boundary $\partial M$, if $h_{t}$ is the second fundamental form of $H_{t}$ for the the metric $g$ in relation to the inward normal $\frac{\partial}{\partial t}$, we get

(i) $\bar{R}\left(X, \frac{\partial}{\partial t}, Y, \frac{\partial}{\partial t}\right)=R\left(X, \frac{\partial}{\partial t}, Y, \frac{\partial}{\partial t}\right)-\frac{q^{\prime}}{q} h_{t}(X, Y)$;

(ii) $\bar{R}\left(X, Y, Z, \frac{\partial}{\partial t}\right)=R\left(X, Y, Z \frac{\partial}{\partial t}\right)$;

(iii) $\bar{R}(X, Y, U, V)=$

$$
R(X, Y, U, V)-\left(1-q^{2}(t)\right)\left[h_{t}(X, U) \cdot h_{t}(Y, V)-h_{t}(X, V) \cdot h_{t}(Y, U)\right] .
$$

Preliminary observations on the demonstration of the Lemma 6.5. Let $D$ (resp. $\bar{D}$ ) be the Levi-Civita connection associated with the metric $g$ (resp. with the metric $\bar{g}$ ). We shall compare the respective Riemann curvature tensors in the point $\Psi(x, t) \in M_{\frac{a}{2}} \backslash M_{\varepsilon}$. Let $c$ and $\bar{c}$ be the geodesics for the metric $g$ and for the metric $\bar{g}$, both geodesics are normal to $H_{t}$ and pass at point $\Psi(x, t)$. The Gauss Lemma ensures that $c$ and $\bar{c}$ are orthogonal to every hypersurface $H_{s}$. Let $\alpha$ be the re-parametrization of the goedesic given by $\alpha(0)=0$ and $\dot{\alpha}(s)=q(\alpha(s))$, since $c(s)=\Psi(x, s)$, we have

$$
\bar{c}(s)=c(\alpha(s))=\Psi(\alpha(s), x) \quad \text { and } \quad \dot{\bar{c}}=q(\alpha(s)) \cdot \dot{c}(\alpha(s)) .
$$


Let $y$ be a vectorial field on $\partial M$, if $m \in \partial M$ is a point of $\partial M$, by means of the parallel transport along the generatrix $t \mapsto(m, t)$ we get a second vector field on the cylinder $\partial M \times[0, a[$, still noted with abuse of notation as $y$. Considering the field $Y=\Psi_{*}(y)$ on $M_{a}$, consequently the map $\left.t \mapsto Y\right|_{(c(t))}$ is a Jacobi field along the geodesic $c$, still noted by $Y$, with abuse of notation. The map $\left.t \mapsto Y\right|_{(\bar{c}(t))}$ is a Jabobi field along the geodesic $\bar{c}$, in fact $Y$ is the field $\frac{\partial \bar{H}}{\partial s}$ along $\bar{c}$ given by the variation $\bar{g}$ - geodesic $\bar{H}(t, s)=\Psi(\alpha(t), x(s))$, where $x^{\prime}(0)=y$. We get:

$$
D_{\dot{c}} D_{\dot{c}} Y+R(Y, \dot{c}) \dot{c}=0 \quad \text { and } \quad \bar{D}_{\dot{\bar{c}}} \bar{D}_{\dot{\bar{c}}} Y+\bar{R}(Y, \dot{\bar{c}}) \dot{\bar{c}}=0 .
$$

We emphasize that

$$
\bar{D}_{\frac{\partial}{\partial t}} Y=D_{\frac{\partial}{\partial t}} Y
$$

in fact $\left[Y, \frac{\partial}{\partial t}\right]=0, g\left(Y, \frac{\partial}{\partial t}\right)=\bar{g}\left(Y, \frac{\partial}{\partial t}\right)=0$ and $g\left(D_{Y} \frac{\partial}{\partial t}, \frac{\partial}{\partial t}\right)=\frac{1}{2} Y$. $g\left(\frac{\partial}{\partial t}, \frac{\partial}{\partial t}\right)$ and $\bar{g}\left(\bar{D}_{Y} \frac{\partial}{\partial t}, \frac{\partial}{\partial t}\right)=\frac{1}{2} Y \cdot \bar{g}\left(\frac{\partial}{\partial t}, \frac{\partial}{\partial t}\right)$. For every vector $Z$ on $\partial M$ we get

$$
\frac{d}{d t} g_{t}(Y, Z)=\frac{\partial}{\partial t} g(Y, Z)=g\left(D_{\frac{\partial}{\partial t}} Y, Z\right)+g\left(Y, D_{\frac{\partial}{\partial t}} Z\right)=2 g\left(D_{\frac{\partial}{\partial t}} Y, Z\right)
$$

for the symmetry of the second fundamental form. In the same way

$$
\frac{d}{d t} g_{t}(Y, Z)=\frac{\partial}{\partial t} \bar{g}(Y, Z)=2 \bar{g}\left(\bar{D}_{\frac{\partial}{\partial t}} Y, Z\right)=2 g\left(\bar{D}_{\frac{\partial}{\partial t}} Y, Z\right)
$$

because on $H_{t}, g$ and $\bar{g}$ are coincident and $\bar{D}_{\frac{\partial}{\partial t} Y}$ and $Z$ are tangent to $H_{t}$. We get

$$
\bar{D}_{\frac{\partial}{\partial t}} Y=D_{\frac{\partial}{\partial t}} Y \quad \text { and } \quad \bar{D}_{Y} \frac{\partial}{\partial t}=D_{Y} \frac{\partial}{\partial t} .
$$

If $f$ is any $C^{1}$ function, replacing $Y$ by $f \cdot Y$ the previous result still remains true, we have indeed

$$
\bar{D}_{\frac{\partial}{\partial t}}(f Y)=\frac{\partial f}{\partial t} \cdot Y+f \cdot \bar{D}_{\frac{\partial}{\partial t}} Y=D_{\frac{\partial}{\partial t}}(f Y) .
$$

As a consequence, every vectorial field $\xi$ such that, for every $(x, t), \xi_{\Psi(x, t)}$ is tangent to $H_{t}$, results in 


$$
\bar{D}_{\frac{\partial}{\partial t}} \xi=D_{\frac{\partial}{\partial t}} \xi \quad \text { and } \quad \bar{D}_{\frac{\partial}{\partial t}}\left(\bar{D}_{\frac{\partial}{\partial t}} \xi\right)=\bar{D}_{\frac{\partial}{\partial t}}\left(D_{\frac{\partial}{\partial t}} \xi\right)=D_{\frac{\partial}{\partial t}}\left(D_{\frac{\partial}{\partial t}} \xi\right) \text {. }
$$

The field $\xi=q(t) \cdot \frac{\partial}{\partial t}$ on $M_{\frac{a}{2}}$ satisfies $\xi_{\bar{c}(s)}=\dot{\bar{c}}(s)$ and yields

$$
\bar{D}_{\dot{\bar{c}}} \bar{D}_{\dot{\bar{c}}} Y=\bar{D}_{q(t) \frac{\partial}{\partial t}} \bar{D}_{q(t) \frac{\partial}{\partial t}} Y=q^{2}(t) D_{\frac{\partial}{\partial t}} D_{\frac{\partial}{\partial t}} Y+q(t) q^{\prime}(t) D_{\frac{\partial}{\partial t}} Y .
$$

Proof of (i) follows directly from the preliminary considerations: since $D_{\dot{c}} Y$ and $R(Y, \dot{c}) \dot{c}$ are tangent to $H_{t}$ we get

$$
\begin{aligned}
\bar{R}(X, \dot{\bar{c}}, Y, \dot{\bar{c}}) & =\bar{g}(\bar{R}(Y, \dot{\bar{c}}) \dot{\bar{c}}, X)=\bar{g}\left(-D_{\dot{\bar{c}}} D_{\dot{\bar{c}}} Y, X\right) \\
& =\bar{g}\left(-q q^{\prime} \cdot D_{\dot{c}} Y, X\right)+\bar{g}\left(-q^{2} D_{\dot{c}} D_{\dot{c}} Y, X\right) \\
& =g\left(-q q^{\prime} \cdot D_{Y} \dot{c}, X\right)+g(R(Y, \dot{c}) \dot{c}, X) \cdot q^{2} \\
& =-q^{\prime} q g\left(D_{Y} \frac{\partial}{\partial t}, X\right)+q^{2} \cdot R\left(X, \frac{\partial}{\partial t}, Y, \frac{\partial}{\partial t}\right) .
\end{aligned}
$$

Proof of (ii). Since $\left[\frac{\partial}{\partial t}, X\right]=0$ and $\bar{D}_{\dot{c}} Y=D_{\dot{c}} Y$, recalling that $D_{\dot{c}} Y$ and $Z$ are tangent to $H_{t}$, we have:

$$
\begin{gathered}
\bar{R}(X, \dot{c}, Z, Y)=\bar{g}\left(\bar{D}_{X} \bar{D}_{\dot{c}} Y, Z\right)-\bar{g}\left(\bar{D}_{\dot{c}} \bar{D}_{X} Y, Z\right) \\
=X \cdot \bar{g}\left(\bar{D}_{\dot{c}} Y, Z\right)-\bar{g}\left(\bar{D}_{\dot{c}} Y, \bar{D}_{X} Z\right)-\frac{\partial}{\partial t} \cdot \bar{g}\left(\bar{D}_{X} Y, Z\right)+\bar{g}\left(\bar{D}_{X} Y, \bar{D}_{\dot{c}} Z\right) \\
=X \cdot g\left(D_{\dot{c}} Y, Z\right)-g\left(D_{\dot{c}} Y,\left(\bar{D}_{X} Z\right)^{\top}\right)-\frac{\partial}{\partial t} \cdot g\left(\left(\bar{D}_{X} Y\right)^{\top}, Z\right)+g\left(\left(\bar{D}_{X} Y\right)^{\top}, D_{\dot{c}} Z\right),
\end{gathered}
$$

being ( $)^{\top}$ the tangential component to $H_{t}$. The metrics $g$ and $\bar{g}$ are coincident on $H_{t}$ and they induce the same connection $D^{T}$ :

$$
\left(\bar{D}_{X} Z\right)^{\top}=D_{X}^{T} Z=\left(D_{X} Z\right)^{\top} .
$$

As a consequence we obtain

$$
\begin{gathered}
\bar{R}(X, \dot{c}, Z, Y)= \\
X \cdot g\left(D_{\dot{c}} Y, Z\right)-\frac{\partial}{\partial t} \cdot g\left(D_{X} Y, Z\right)+g\left(D_{X} Y, D_{\dot{c}} Z\right)-g\left(D_{\dot{c}} Y ; D_{X} Z\right)= \\
R(X, \dot{c}, Z, Y)
\end{gathered}
$$

proving the (ii) equality.

Proof of (iii). This equality follows from Gauss's Theorema Egreguim. Let $R_{t}$ be the Riemann curvature tensor induced by the metric $g$ on the hypersurface $H_{t}$. On $H_{t}$ we have $g=\bar{g}$ and consequently 
ESTIMATES OF THE LAPLACIAN SPECTRUM AND BOUNDS OF

$$
R_{t}(X, Y, U, V)=R(X, Y, U, V)+h_{t}(X, U) \cdot h_{t}(Y, V)-h_{t}(X, V) \cdot h_{t}(Y, V)
$$

and

$$
R_{t}(X, Y, U, V)=\bar{R}(X, Y, U, V)+\bar{h}_{t}(X, U) \cdot \bar{h}_{t}(Y, V)-\bar{h}_{t}(X, V) \cdot \bar{h}_{t}(Y, V)
$$

being $\bar{h}_{t}$ the second fundamental form of the hypersurface $H_{t}$ for the metric $\bar{g}$ :

$$
\bar{h}_{t}(X, Y)=\bar{g}\left(\bar{D}_{X} a \frac{\partial}{\partial t}, Y\right)=\bar{g}\left(\bar{D}_{q \cdot \dot{c}} X, Y\right)=q \cdot h_{t}(X, Y)
$$

because $\bar{D}_{\dot{c}} X=D_{\dot{c}} X$ is tangent to $H_{t}$ and $\left[q \frac{\partial}{\partial t}, X\right]=0$. We get

$$
\begin{gathered}
\bar{R}(X, Y, U, V)=R_{t}(X, Y, U, V)-q^{2}\left[h_{t}(X, U) \cdot h_{t}(Y, V)-h_{t}(X, V) \cdot h_{t}(Y, u)\right] \\
=R(X, Y, U, V)+\left(1-q^{2}\right)\left[h_{t}(X, U) \cdot h_{t}(Y, V)-h_{t}(X, V) \cdot h_{t}(Y, u)\right]
\end{gathered}
$$

We are now able to compare the sectional curvatures, this is done in the following

Lemma 6.6. With the same notations as Lemma 6.5, let $\bar{\sigma}$ (resp. $\sigma$ ) be the sectional curvature, at distance $t$ from the boundary $\partial M$, for the metric $\bar{g}$ (resp. g). If $h_{\min }(t)$ and $h_{\max }(t)$ are respectively the minimal and maximal eigenvalues of the second fundamental form of the hypersurface $H_{t}$ for the metric $g$, we have

$$
\begin{gathered}
\left.\left.\bar{\sigma} \geq \min \left\{\left(1-q^{2}(t)\right) \cdot h_{\max }^{2}(t)\right),\left(1-q^{2}(t)\right) \cdot h_{\min }^{2}(t)\right), h_{\max }(t)\left(1-q^{2}(t)\right) \cdot h_{\min }(t), 0\right\} \\
+\min \left\{q^{2}(t) \cdot \sigma, \sigma\right\}-2 \max \left\{q^{\prime}(t) q(t) h_{\max }(t), q^{\prime}(t) q(t) h_{\min }(t), 0\right\} .
\end{gathered}
$$

Proof. The joint use of previous Lemma and the Riemann curvature tensor symmetry properties give, for all real constants $\alpha, \beta$ :

$$
\bar{R}\left(X+\alpha \cdot q(t) \cdot \frac{\partial}{\partial t}, Y+\beta \cdot q(t) \cdot \frac{\partial}{\partial t}, X+\alpha \cdot q(t) \cdot \frac{\partial}{\partial t}, Y+\beta \cdot q(t) \cdot \frac{\partial}{\partial t}\right)=
$$


ESTIMATES OF THE LAPLACIAN SPECTRUM AND BOUNDS OF

TOPOLOGICAL INVARIANTS FOR RIEMANNIAN MANIFOLDS WITH

BOUNDARY.

$$
\begin{aligned}
& R\left(X+\alpha \cdot q(t) \cdot \frac{\partial}{\partial t}, Y+\beta \cdot q(t) \cdot \frac{\partial}{\partial t}, X+\alpha \cdot q(t) \cdot \frac{\partial}{\partial t}, Y+\beta \cdot q(t) \cdot \frac{\partial}{\partial t}\right)+ \\
+ & \left(1-q^{2}(t)\right)\left[h_{t}(X, X) \cdot h_{t}(Y, Y)-h_{t}^{2}(X, Y)\right]-q^{\prime}(t) q(t) h_{t}(\beta X-\alpha Y, \beta X-\alpha Y) .
\end{aligned}
$$

On a $n$-dimensional Euclidean space, let $h$ be any symmetric bilinear form whose eigenvalues are $\eta_{i}$; in an orthonormal eigenvectors base $\left\{\mathbf{e}_{i}\right\}_{i=1, \ldots, n}$ we have

$$
X=\sum_{i=1}^{k} x_{i} \cdot \mathbf{e}_{i} \quad \text { and } \quad Y=\sum_{i=1}^{k} y_{i} \cdot \mathbf{e}_{i}
$$

and

$$
\begin{aligned}
h(X, X) \cdot h(Y, Y)-h^{2}(X, Y)= & \left(\sum_{i=1}^{n} \eta_{i} \cdot x_{i}^{2}\right) \cdot\left(\sum_{j=1}^{n} \eta_{j} \cdot y_{j}^{2}\right)-\left(\sum_{k, l=1}^{n} \eta_{k} \eta_{l} x_{k} x_{l}\right)^{2}= \\
& \sum_{i<j} \eta_{i} \eta_{j}\left(x_{i} y_{j}-x_{j} y_{i}\right)^{2} .
\end{aligned}
$$

If $X+\alpha q \dot{c}$ and $Y+\beta q \dot{c}$ are $\bar{g}$-orthogonals, we get:

$$
\begin{aligned}
1 & =\bar{g}(X+\alpha q \dot{c}, X+\alpha a \dot{c},) \cdot \bar{g}(Y+\beta q \dot{c}, Y+\beta q \dot{c})-\bar{g}(X+\alpha q \dot{c}, Y+\beta q \dot{c})^{2} \\
& =\left[g(X, X)+\alpha^{2}\right] \cdot\left[g(Y, Y)+\beta^{2}\right]-[g(X, Y)+\alpha \beta] \\
& =g(X, X) \cdot g(Y, Y)-g(X, Y)^{2}+g(\beta X-\alpha Y, \beta X-\alpha Y) .
\end{aligned}
$$

and as a consequence

$$
0 \leq g(X, X) \cdot g(Y, Y)-g(X, Y)^{2} \leq 1 \quad 0 \leq g(\beta X-\alpha Y, \beta X-\alpha Y) \leq 1 .
$$

Moreover, since the $n-1$ relative eigenvalues of $g$ to $\bar{g}$ are equal to 1 except one which is equal to $q^{2}$, we get

- if $q(t)<1$,

$$
\begin{gathered}
q^{2}(t) \leq g(X+\alpha q \dot{c}, X+\alpha q \dot{c},) \cdot g(Y+\beta q \dot{c}, Y+\beta q \dot{c},) \\
-g(X+\alpha q \dot{c}, Y+\beta q \dot{c},)^{2} \leq 1
\end{gathered}
$$

- if $q(t) \geq 1$,

$$
\begin{gathered}
1 \leq g(X+\alpha q \dot{c}, X+\alpha q \dot{c},) \cdot g(Y+\beta q \dot{c}, Y+\beta q \dot{c},) \\
-g(X+\alpha q \dot{c}, Y+\beta q \dot{c},)^{2} \leq q^{2}(t)
\end{gathered}
$$


ESTIMATES OF THE LAPLACIAN SPECTRUM AND BOUNDS OF

and

$$
\begin{gathered}
h(X, X) \cdot h(Y, Y)^{2}-h(X, Y)^{2} \\
\leq \sup \left\{h_{\text {min }}^{2}, h_{\text {max }}^{2}\right\}\left[h(X, X) \cdot h(Y, Y)^{2}-h(X, Y)^{2}\right], \\
h(X, X) \cdot h(Y, Y)^{2}-h(X, Y)^{2} \\
\geq \inf \left\{h_{\text {min }}^{2}, h_{\text {max }}^{2}, h_{\text {min }} h_{\max }, 0\right\}\left[h(X, X) \cdot h(Y, Y)^{2}-h(X, Y)^{2}\right], \\
h_{\text {min }} \cdot g(\beta X-\alpha Y, \beta X-\alpha Y) \leq h(\beta X-\alpha Y, \beta X-\alpha Y) \\
\leq h_{\text {max }} \cdot g(\beta X-\alpha Y, \beta X-\alpha Y) .
\end{gathered}
$$

All the combined results prove the Lemma.

We now have all the tools to prove all the results concerning the control of the curvature and consequently the first main result of this section.

Main Theorem 6.7. Let $(M, \partial M)$ be a Riemannian manifold with convex boundary, then there exists a sequence of $C^{\infty}$-metrics $\left\{g_{k}\right\}_{k \in \mathbb{N}}$ on $M \sharp M$ and converging in the $C^{0}$ topology to $g \sharp g$, such that

$$
\min \left(\sigma_{g_{k}}\right) \geq\left(\sigma_{g}\right)
$$

where the minimum is achieved on all the 2-planes tangent to $M$.

Proof. Let $a$ be the injectivity radius of the boundary and let $H_{t}=$ $\{x \in M$ s.t. $d(X, \partial M)=t\}$ be the hypersurface of $M$ at $t$-distance from the boundary $\partial M$. It is assumed that its second fundamental form is negative definite for all $t \leq \frac{a}{2}$.

Let $\phi_{\varepsilon}$ be the function $\left.\left.\left.\left.\phi_{\varepsilon}:\right] 0,+\infty\right] \rightarrow\right] 0,+\infty\right], 0<\varepsilon \leq \frac{a}{2}$, enjoying the properties p.1 - p.5 (see p. 199); for all $t \in[0, a[$ the corresponding metric splits as $g=d t^{2} \oplus g_{\phi_{\varepsilon}}$. Based on these assumptions, we obtain

$$
q^{2}(t)=\left[\phi_{\varepsilon}^{\prime}\left(\phi_{\varepsilon}^{-1}(t)\right)\right]^{2} \leq 1
$$

and

$$
q(t) \cdot q^{\prime}(t)=\phi_{\varepsilon}^{\prime}\left(\phi_{\varepsilon}^{-1}(t)\right) \cdot \phi_{\varepsilon}^{\prime \prime}\left(\phi_{\varepsilon}^{-1}(t)\right) \cdot \frac{1}{\phi_{\varepsilon}^{\prime}\left(\phi_{\varepsilon}^{-1}(t)\right)}=\phi_{\varepsilon}^{\prime \prime}\left(\phi_{\varepsilon}^{-1}(t)\right) \geq 0 .
$$


ESTIMATES OF THE LAPLACIAN SPECTRUM AND BOUNDS OF

Making use of the (6.2) formula of Lemma 6.6 we get

$$
\begin{aligned}
& \bar{R}\left(X+\alpha \cdot q(t) \cdot \frac{\partial}{\partial t}, Y+\beta \cdot q(t) \cdot \frac{\partial}{\partial t}, X+\alpha \cdot q(t) \cdot \frac{\partial}{\partial t}, Y+\beta \cdot q(t) \cdot \frac{\partial}{\partial t}\right) \\
= & R\left(X+\alpha \cdot q(t) \cdot \frac{\partial}{\partial t}, Y+\beta \cdot q(t) \cdot \frac{\partial}{\partial t}, X+\alpha \cdot q(t) \cdot \frac{\partial}{\partial t}, Y+\beta \cdot q(t) \cdot \frac{\partial}{\partial t}\right) \\
+ & \left(1-q^{2}(t)\right)\left[h_{t}(X, X) h_{t}(Y, Y)-h_{t}^{2}(X, Y)\right]-q^{\prime}(t) \cdot q(t) \cdot h_{t}(\beta X-\alpha Y, \beta X-\alpha Y) \\
\geq & \bar{R}\left(X+\alpha \cdot q(t) \cdot \frac{\partial}{\partial t}, Y+\beta \cdot q(t) \cdot \frac{\partial}{\partial t}, X+\alpha \cdot q(t) \cdot \frac{\partial}{\partial t}, Y+\beta \cdot q(t) \cdot \frac{\partial}{\partial t}\right)
\end{aligned}
$$

and because $h_{\max }(t)$ and $h_{\min }(t)$ are both negative and $h_{\max }(t) \cdot h_{\min }(t)>$ 0 , we showed that, for every $\varepsilon<a, \min \left(\sigma_{g_{\phi_{\varepsilon}}}\right) \geq \min \left(\sigma_{g}\right)$. The $t \mapsto \phi_{\varepsilon}-t$ map is a decreasing function; and consequently we get $0 \leq \phi_{\varepsilon}-t \leq \varepsilon$ for all $t \in\left[0, a\right.$ [ and also that $g_{\phi_{\varepsilon}}$ is close to $g$ for the $C^{0}$-topology. We conclude the proof sending $\varepsilon$ to $0^{+}$.

Lemma 6.8. Let $(M, \partial M, g)$ be a compact Riemannian manifold with boundary and $\eta>0, \delta$ and $k$ three real numbers such that

$$
h_{t, \partial M}<\eta, \quad \quad \text { Ricci }_{g} \geq-(n-1) \cdot \delta^{2} \cdot g, \quad \sigma_{g} \geq-k^{2} ;
$$

then, for every $0<\varepsilon<\frac{a}{2}$ we have:

(i) the assumption Ricci $_{g} \geq-(n-1) \cdot \delta^{2} \cdot g$ implies that

$$
d v_{\tilde{g}} \leq\left[\cosh (\delta \varepsilon)+\sup \left(1, \frac{\eta}{\delta}\right) \cdot \sinh (\delta \varepsilon)\right] d v_{g}
$$

;

(ii) the assumption $\sigma_{g} \geq-k^{2}$ implies that

$$
\begin{gathered}
\left(\frac{\sinh \left[k\left(\frac{a}{2}-\varepsilon\right)\right]}{\sinh \left(\frac{k a}{2}\right)}\right)^{2} \cdot g \\
\leq \tilde{g} \leq\left[\cosh (\delta \varepsilon)+\sup \left(1, \frac{\eta}{\delta}\right) \cdot \sinh (\delta \varepsilon)\right]^{2 n-2} \cdot \frac{\left[\sinh \left(\frac{k a}{2}\right)\right]^{2 n-4}}{\left[\sinh \left(k\left(\frac{a}{2}-\varepsilon\right)\right)\right]^{2 n-4}} \cdot g .
\end{gathered}
$$


ESTIMATES OF THE LAPLACIAN SPECTRUM AND BOUNDS OF TOPOLOGICAL INVARIANTS FOR RIEMANNIAN MANIFOLDS WITH BOUNDARY.

Proof of (i). Let $c$ be the geodesic normal to the boundary. At point $c(t)=(x, t)$ the volume forms for the metrics $g$ and $\tilde{g}$ are given respectively by $d v_{g}=\vartheta(t) d x d t$ and $d v_{\tilde{g}}=\vartheta(\phi(t)) d x d t$. Given that the Bishop Comparison Theorem assures that $\tilde{\vartheta}(t)=\left[\cosh (\delta t)+\frac{\eta}{\delta} \sinh (\delta t)\right]^{n-1}$ and that the function $\frac{\vartheta}{\tilde{\vartheta}}$ is decreasing we deduce that

$$
\frac{\vartheta[\phi(t)]}{\vartheta(t)} \leq \frac{\tilde{\vartheta}[\phi(t)]}{\tilde{\vartheta}(t)} \leq\left[\frac{\cosh (\delta(t+\varepsilon))+\frac{\eta}{\delta} \sinh (\delta(t+\varepsilon))}{\cosh (\delta t)+\frac{\eta}{\delta} \sinh (\delta t)}\right]^{n-1} .
$$

We replace $\frac{\eta}{\delta}$ on the left side of the previous inequality chain with $\sup \left[1, \frac{\eta}{\delta}\right]$ obtaining a decreasing function which attains its minimum in $t=0$, proving the inequality in (i).

Proof of (ii). Let $\mathbb{J}$ be the set of all Jacobi fields normal to the geodesic $c$, i.e. $J \in \mathbb{J} \Leftrightarrow g\left(J^{\prime}(t), c^{\prime}(t)\right)=0$ for all $t$. At point $c(t)=(x, t)$ we have

$$
\tilde{g} \geq \inf _{J \in \mathbb{J}} \frac{g(J(\phi(t)), J(\phi(t)))}{g(J(t), g(J(t))} \cdot g .
$$

Vector $J$ is indeed the image of a vector $X$, tangent to $\partial M$ via the tangent map to the chart $\Psi: \partial M \times] 0, a\left[\rightarrow M, \Psi(x, t)=\exp _{x}(t \cdot N(x))\right.$. We consider the vector field $\bar{J}(t)=\sinh [k(a-t)] \mathbf{u}, \mathbf{u}$ being a unit vector field normal to $\bar{c}$ in the reference space of sectional curvature identically equal to $-k^{2}$. We get

$$
\begin{gathered}
\frac{g(J[\phi(t)], J[\phi(t)])}{g(J(t), J(t))} \geq \frac{g(J[\phi(t)], J[\phi(t)])}{\bar{g}(\bar{J}(t), \bar{J}(t))} \\
\geq \frac{\bar{g}(\bar{J}[\phi(t)], \bar{J}[\phi(t)])}{\bar{g}(\bar{J}(t), \bar{J}(t))} \geq \frac{\bar{J}^{2}(t+\varepsilon)}{\bar{J}^{2}(t)} \geq \frac{\bar{J}^{2}\left(\frac{a}{2}-\varepsilon\right)}{\bar{J}^{2}\left(\frac{a}{2}\right)},
\end{gathered}
$$

where the first inequality comes from Rauch Comparison Theorem, while the others derive from the fact that function $\frac{|J|}{\bar{J}}$ is a decreasing function, (see the proof of Lemma 6.1). This chain of inequalities proves the left inequality of (ii). Let $\left\{\mu_{i}\right\}_{i=1, \ldots n}$ be the relative eigenvalues of $\tilde{g}$ to $g$; we have $\mu_{1}=1$ and $\mu_{i} \geq\left(\frac{\sinh \left[k\left(\frac{a}{2}-\varepsilon\right)\right]}{\sinh k\left(\frac{a}{2}\right)}\right)$ for $i \geq 2$; from what has just been proved in (i) we have $\prod_{i=2}^{n} \mu_{i} \geq\left[\cosh (\delta t)+\frac{\eta}{\delta} \sinh (\delta t)\right]^{2 n-2}$. This inequality shows an upper bound of each eigenvalue $\mu_{i}$ and consequently proves the inequalities chain in (ii). 
ESTIMATES OF THE LAPLACIAN SPECTRUM AND BOUNDS OF

Main Theorem 6.9. Let $(M, \partial M, g)$ be a Riemannian manifold with nonempty boundary and let $k, a$ and $\eta$ be three real numbers such that

$$
\sigma_{g} \geq-k^{2}, \quad \quad \operatorname{inj}_{\partial M} \geq a>0, \quad h_{\partial M} \leq \eta .
$$

Then there exists a metric $\tilde{g}$ on $(M, \partial M)$ such that $\tilde{g} \sharp \tilde{g}$ is $C^{\infty}$ on $M \sharp M$ and such that

$$
\begin{gathered}
\left(\frac{\sinh \left(\frac{k a}{4}\right)}{\sinh \left(\frac{k a}{2}\right)}\right)^{2} \cdot g \leq \tilde{g} \\
\leq\left[\cosh \left(\frac{k a}{4}\right)+\sup \left(1, \frac{\eta}{k}\right) \cdot \sinh \left(\frac{k a}{4}\right)\right]^{2 n-2} \cdot\left(\frac{\sinh \left(\frac{k a}{2}\right)}{\sinh \left(\frac{k a}{4}\right)}\right)^{2 n-4} \cdot g
\end{gathered}
$$

and

$$
\sigma_{\tilde{g}} \geq-\frac{4 k^{2}}{\tanh ^{2}\left(\frac{k a}{4}\right)}
$$

Proof. The (6.4) inequalities are easily derived from (6.3) inequalities setting $\varepsilon=\frac{a}{4}$.

If the boundary $\partial M$ is convex, the (6.5) inequality arises from Proposition 6.7 :

$$
\bar{R}(X, Y, X, Y) \geq-k^{2} \cdot g(X, Y) \geq-\frac{4 k^{2}}{\tanh ^{2}\left(\frac{k a}{4}\right)} \cdot g(X, Y)
$$

On the contrary, if the boundary is not convex, we set $l=\frac{a}{4}$ and we consider the function $\phi_{\frac{a}{4}}:[0,+\infty[\rightarrow[0,+\infty[$ enjoying as usual the properties p.1 and p.2 (see p.199). To do this, it is necessary to have $\phi_{\frac{a}{4}}^{\prime}\left(\frac{a}{2}\right)=1$ and thus $\int_{0}^{\frac{a}{2}} \phi_{\frac{a}{4}}{ }^{\prime \prime}(s) d s=1$. Its second derivative $\phi_{\frac{a}{4}}{ }^{\prime \prime}$ has to satisfy the following properties:

- $\phi_{\frac{a}{4}}{ }^{\prime \prime} \in C^{\infty}([0,+\infty[)$;

- the support of $\phi_{\frac{a}{4}}{ }^{\prime \prime}$ is compact and also contained in the open $] 0, \frac{a}{2}[$;

- $\int_{0}^{\frac{a}{2}} \phi_{\frac{a}{4}}{ }^{\prime \prime}(t) d t=1$;

- $0 \leq \phi_{\frac{a}{4}}{ }^{\prime \prime}(t) \leq \frac{4}{a}$ and 
ESTIMATES OF THE LAPLACIAN SPECTRUM AND BOUNDS OF

TOPOLOGICAL INVARIANTS FOR RIEMANNIAN MANIFOLDS WITH

BOUNDARY.

- its graphic must be symmetric with the right $x-\frac{a}{4}=0$.

It follows that $\phi_{\frac{a}{4}}^{\prime}\left(\frac{a}{2}-s\right)+\phi_{\frac{a}{4}}^{\prime}(s)=1$ and that $\int_{0}^{\frac{a}{2}} \phi_{\frac{a}{4}}^{\prime}(s) d s=\frac{a}{4}$; so $\phi_{\frac{a}{4}}(t)=$ $\frac{a}{4}+\int_{0}^{t} \phi_{\frac{a}{4}}^{\prime}(s) d s$ remains. From Proposition 6.4 it follows that on $M \backslash M_{\frac{a}{4}}$ the metric $\tilde{g}=d t^{2} \oplus g_{\phi(t)}$ is isometric to the metric $\frac{d t^{2}}{\left[\phi_{\frac{a}{4}}^{\prime}\left(\phi_{\frac{a}{4}}^{-1}(t)\right)\right]^{2}} \oplus g_{t}$. Moreover we emphasize here that, for every $t$, we have

$$
\begin{aligned}
& 0 \leq \quad \phi_{\frac{a}{4}}^{\prime}(t) \quad \leq 1 \\
& 0 \leq a(t)=\phi_{\frac{a}{4}}^{\prime}\left(\phi_{\frac{a}{4}}^{-1}(t)\right) \leq 1 \\
& 0 \leq a(t) \cdot q^{\prime}(t)={\phi_{\frac{a}{4}}^{\prime}}^{\prime}\left(\phi_{\frac{a}{4}}^{-1}(t)\right) \leq \frac{4}{a} .
\end{aligned}
$$

Exploiting the results of Lemma 6.6 we get

$$
\bar{\sigma} \geq \sigma+\left(1-q^{2}(t)\right) h_{\max } h_{\min }-2 q(t) \cdot q^{\prime}(t) h_{\max } .
$$

The use now of Lemma 6.1 yields for every $t \in\left[\frac{a}{4}, \frac{a}{2}\right]$

$$
h_{\max } \leq \frac{k}{\tanh \left(\frac{k a}{4}\right)} \quad \text { and } \quad h_{\min } \cdot h_{\max } \geq-\frac{k^{2}}{\tanh \left(\frac{k a}{4}\right) \cdot \tanh \left(\frac{k a}{2}\right)}
$$

Remembering that $\tanh x \leq x$, putting the (6.7) inequalities in the (6.6) we achieve,

$$
\bar{\sigma} \geq-\frac{4 k^{2}}{\tanh ^{2}\left(\frac{k a}{4}\right)}
$$

which ends the proof of the Theorem.

\section{References}

[1] M. Berger, P. Gauduchon, E. Mazet, Le spectre d'une variété riemannienne, Lecture notes in Maths 194, Springer (1987).

[2] M. P. do Carmo, Riemannian Geometry, Birkäuser, Boston (1992);

[3] S. Gallot: A Sobolev inequality and some geometric applications, Spectra of Riemannian manifolds, Kaigai Publication, Tokyo (1983), pp. 45-55. 
ESTIMATES OF THE LAPLACIAN SPECTRUM AND BOUNDS OF TOPOLOGICAL INVARIANTS FOR RIEMANNIAN MANIFOLDS WITH BOUNDARY.

[4] S. Gallot: Inégalités isopérimetriques, courbure de Ricci et invariants géométriques I, C. R. Acad. Sci. serie I, 296 (1983), pp. 333-336.

[5] S. Gallot: Inégalités isopérimetriques, courbure de Ricci et invariants géométriques II, C. R. Acad. Sci. serie I, 296 (1983), pp. 365-368.

[6] S. Gallot, D. Hulin, J. La Fontaine, Riemannian Geometry, Springer, Berlin (2007).

[7] M. Gromov: Structures métriques pour les variétés riemanniennes, Textes Math. 1, Cedic-Nathan, (1981).

[8] J. Hersch: Quatre propriétés isopérimétriques de membranes sphériques homogénes, C. R. Acad. Sci. Paris, 270 (1970), pp. 1645-1648.

[9] F. Laudenbach: A Morse Complex for Manifolds with boundary, Geom. Dedicata 153 (2011), pp. 47-57.

[10] P. Li, S. T. Yau: Estimates of eigenvalues of a compact Riemannian manifold, AMS Proc. Symp. Pure Math. 36 (1980), pp. 205-240;

[11] D. Meyer: Minoration de la premiere valeur propre non nulle du problème de Neumann sur les variétés riemanniens à bord, Ann. Inst. Fouruer, Grenoble 36, 2 (1986), pp.113-125;

[12] L. E. Payne and H. F. Weinberger: Lower bounds for vibration frequencies of elastically supported membranes and plates, J. Soc. Ind. Appi. Math. 5 (1957), pp. 171-182.

[13] L. Sabatini: Estimation of Vibration Frequencies of Linear Elastic Membranes, Applications of Mathematics 63 (2018), pp. 3753 ;

[14] P. Yang, S. T. Yau: Eigenvalues of the laplacian of compact Riemann surfaces and minimal submanifolds, Ann. Scuola N. Sup. Pisa 7 (1980), pp. 55-63

Luca Sabatini,

Dipartminto di Scienze di Base ed Applicate per l'Ingegneria

Università degli Studi di Roma La Sapienza,

Via A. Scarpa 16, 00100 Roma, Italia.

Email: luca.sabatini@sbai.uniroma1.it 